\title{
Integral equations requiring small numbers of Krylov-subspace iterations for two-dimensional smooth penetrable scattering problems
}

\author{
Yassine Boubendir \\ New Jersey Institute of Technology, boubendi@njit.edu \\ Oscar Bruno \\ Caltech,obruno@caltech.edu \\ David Levadoux \\ ONERA, david.levadoux@onera.fr \\ Catalin Turc \\ New Jersey Institute of Technology, catalin.c.turc@njit.edu
}

\begin{abstract}
This paper presents a class of boundary integral equations for the solution of problems of electromagnetic and acoustic scattering by two dimensional homogeneous penetrable scatterers with smooth boundaries. The new integral equations, which, as is established in this paper, are uniquely solvable Fredholm equations of the second kind, result from representations of fields as combinations of single and double layer potentials acting on appropriately chosen regularizing operators. As demonstrated in this text by means of a variety of numerical examples (that resulted from a high-order Nyström computational implementation of the new equations), these "regularized combined equations" can give rise to important reductions in computational costs, for a given accuracy, over those resulting from previous iterative boundary integral equation solvers for transmission problems.
\end{abstract}

Key words: Electromagnetic Scattering, Transmission Problems, Combined Field Integral Equations, Pseudo-differential Operators, Regularizing Operators

1991 MSC: 65R20, 35E99, 45B05,

Preprint submitted to Elsevier

(C) 2015. This manuscript version is made available under the Elsevier user license http://www.elsevier.com/open-access/userlicense/1.0/ 


\section{Introduction}

Owing mainly to the dimensional reduction and the absence of dispersion errors inherent in boundary integral formulations, scattering solvers based on boundary integral equations can be significantly more efficient than solvers resulting from direct discretization of the corresponding Partial Differential Equations (PDE). In view of the large dense systems of linear equations that result from integral equation formulations, however, integral-equation solvers often rely on use of Krylov subspace iterative solvers in conjunction with fast algorithms $[6,11,36]$ for evaluation of matrix vector products. Since the numbers of iterations required by Krylov subspace solvers for a given system of equations depends greatly on the spectral character of the system matrix, the efficiency of boundary iterative integral solvers hinges on the spectral properties of the integral formulations used.

In this paper we introduce a new class of boundary integral equations for the solution of electromagnetic and acoustic transmission problems (that is, problems of diffraction and scattering by penetrable scatterers) for two dimensional homogeneous penetrable scatterers with smooth boundaries. The new formulations, which, as established in this paper, are expressed in terms of uniquely solvable Fredholm equations of the second kind, result from representations of fields as combinations of single and double layer potentials acting on regularizing operators analogous to those used in $[2,4,5,7,9,10,12,13,27$ 29] for non-penetrable scatterers. As demonstrated in this text by means of a variety of numerical examples, for a given accuracy, the new "regularized combined equations" can give rise to significant reductions in iteration numbers, and therefore, in overall computational costs, over those resulting from previous boundary integral formulations. Extensions of the techniques introduced in this paper to non-smooth boundaries and three dimensional problems is currently underway. We are also investigating the possibility of adapting the regularization procedure put forth in this paper to the important case of dielectric materials with junction points that has recently received significant attention in the literature $[14,15,18,34]$.

Uniquely solvable formulations for transmission scattering problems have been available for quite some time [32]. For example, a class of such integral equations results from representation of the fields inside and outside the dielectric scatterer by means of Green's formula: linear combinations of the interior and exterior boundary values of the fields and their normal derivatives can be used to produce various types of pairs of boundary integral equations with two unknown functions, including: (1) A family of integral equations of the second kind (which feature multiples of the identity operator and linear combinations of compact operators, including the single and double layer operators as well as suitable compact differences of hypersingular integral operators [19, 24, 32, 35]); 
and (2) Integral equations of the first kind with positive principal part which include non-compact hypersingular operators. Equations of the type (1) and (2) are used most frequently as part of integral solvers for wave transmission problems.

(It is also possible to express the transmission problem in terms of a single uniquely solvable integral equation; the papers $[20,26]$ present comprehensive discussions in these regards. It is useful to mention here that, while single transmission integral equations contain half as many unknowns as the corresponding systems, they require operator compositions which, in the context of iterative solvers considered in this paper, may lead to equal or even higher computing costs than the system formulations such as those mentioned above.)

This paper presents novel regularized integral equation formulations for the transmission problem. Unlike the second-kind formulations introduced earlier, which rely on cancellation of hypersingular terms in the integral kernels, the present approach produces second-kind formulations via regularization of hypersingular operators by means of suitable regularizing operators $[9,10,12,13$, $28,29]$. In particular, use of regularizing operators given by layer-potentials with complex wavenumbers results in second-kind Fredholm equations with improved spectral properties. Similar regularized equations result from use of regularizing operators expressed in terms of Fourier operators whose symbols have the same high-frequency asymptotics as the corresponding layer-potential regularizers mentioned above.

In order to demonstrate the properties of the new equations we introduce Nyström implementations of the previous and new formulations considered. These implementations, which are based on the methods introduced in $[8$, $22,25,31$ ], include elements such as approximation via global trigonometric polynomials, splitting of integral kernels into singular and smooth components, and explicit quadrature of products of logarithmically singular terms and trigonometric polynomials.

This paper is organized as follows: in Sections 2, 3, and 4 we review the classical systems of integral equations for the solution of transmission scattering problems, we introduce the new regularized formulations, and we establish their unique solvability and second-kind Fredholm character in the appropriate functional spaces. The reductions in iteration numbers and computing times that result from the new formulations are demonstrated in Section 5. 


\section{Acoustic transmission problems}

We consider the problem of evaluation of the time-harmonic fields that arise as an incident field $u^{i n c}$ impinges upon a homogeneous dielectric scatterer which occupies a bounded region $\Omega_{2} \subset \mathbb{R}^{2}$. Calling $\Omega_{1}=\mathbb{R}^{2} \backslash \Omega_{2}$ the region exterior to the obstacle, in what follows $\epsilon_{1}$ and $\epsilon_{2}$ denote the electric permeabilities of the materials in regions $\Omega_{1}$ and $\Omega_{2}$, respectively; the permeabilities of both dielectrics is assumed to equal $\mu_{0}$, the permeability of vacuum. Letting $u^{1}$ and $u^{2}$ denote the scattered field in $\Omega_{1}$ and the total field in $\Omega_{2}$, respectively, calling $\Gamma$ the boundary between the domains $\Omega_{1}$ and $\Omega_{2}$ (which, throughout this text is assumed to be smooth), and given an incident field $u^{\text {inc }}$ for which

$$
\Delta u^{i n c}+k_{1}^{2} u^{i n c}=0 \quad \text { in } \mathbb{R}^{2},
$$

the fields $u^{1}$ and $u^{2}$ can be obtained as the solutions to the Helmholtz equations

$$
\begin{array}{ll}
\Delta u^{1}+k_{1}^{2} u^{1}=0 & \text { in } \Omega_{1}, \\
\Delta u^{2}+k_{2}^{2} u^{2}=0 & \text { in } \Omega_{2},
\end{array}
$$

which satisfy the transmission conditions

$$
\begin{array}{clrl}
u^{1}+u^{i n c} & =u^{2} & \text { on } \Gamma \\
\frac{\partial u^{1}}{\partial n}+\frac{\partial u^{i n c}}{\partial n} & =\nu \frac{\partial u^{2}}{\partial n} & \text { on } \Gamma
\end{array}
$$

as well as the Sommerfeld radiation condition at infinity:

$$
\lim _{|r| \rightarrow \infty} r^{1 / 2}\left(\partial u^{1} / \partial r-i k_{1} u^{1}\right)=0 .
$$

In these equations $n$ denotes the unit normal on $\Gamma$ that points into $\Omega_{2}$ and $k_{j}$ denotes the wavenumber in the region $\Omega_{j}(j=1,2)$; for a given frequency $\omega$ we have $k_{i}=\omega \sqrt{\mu_{0} \epsilon_{j}}$. The constant $\nu$ in (4), finally, is given by $\nu=1$ under $E$-polarization and $\nu=\epsilon_{1} / \epsilon_{2}$ under $H$-polarization.

Remark 2.1 As is well known, the transmission problem (2)-(5) admits a unique solution provided the wavenumbers $k_{j}, j=1,2$ are either real numbers or complex numbers satisfying certain algebraic conditions [20, p. 309]; see also [24]. In this text it is assumed that either $k_{j}, j=1,2$ are real numbers or, otherwise, that equations (2)-(5) admit unique solutions.

\section{Classical boundary integral formulations}

A variety of integral equation formulations for the transmission problem (2)(5) exist (e.g. [17, 20,24,32]). In the spirit of the general boundary integral 
method, these formulations express the solution of equation (2)-(5) in terms of boundary integral representations that include free-space Green functions and surface potentials as well as unknown functions defined on the boundary $\Gamma$ (the so-called "integral densities"); the unknown densities, in turn, are determined as solutions of corresponding integral equations.

The two-dimensional free-space Green function $G_{k}$ for a wavenumber $k$ is given by

$$
G_{k}(\mathbf{x})=\frac{i}{4} H_{0}^{(1)}(k|\mathbf{x}|)
$$

Given a wavenumber $k$, the single and double layer potentials for the wavenumber $k$ and with respective densities $\varphi$ and $\psi$ are functions defined throughout $\mathbb{R}^{2} \backslash \Gamma$ by means of the integral expressions

$$
S_{k}[\varphi](\mathbf{z})=\int_{\Gamma} G_{k}(\mathbf{z}-\mathbf{y}) \varphi(\mathbf{y}) d s(\mathbf{y}), \quad \mathbf{z} \in \mathbb{R}^{2} \backslash \Gamma \quad \text { (Single Layer Potential) }
$$

and

$$
D_{k}[\psi](\mathbf{z})=\int_{\Gamma} \frac{\partial G_{k}(\mathbf{z}-\mathbf{y})}{\partial n(\mathbf{y})} \psi(\mathbf{y}) d s(\mathbf{y}), \mathbf{z} \in \mathbb{R}^{2} \backslash \Gamma \quad \text { (Double Layer Potential). }
$$

Clearly $S_{k}[\varphi]$ and $D_{k}[\psi]$ are solutions of the Helmholtz equation $\Delta \psi+k^{2} \psi=0$ in $\mathbb{R}^{2} \backslash \Gamma$.

The Green identities are often utilized in derivations of integral equation formulations; in the present context they can be used to express the solutions $u_{1}$ and $u_{2}$ in terms of single and double layer potentials on $\Gamma$ :

$$
u^{1}(\mathbf{z})=D_{k_{1}}\left[u^{1}\right](\mathbf{z})-S_{k_{1}}\left[\frac{\partial u^{1}}{\partial n}\right](\mathbf{z}), \mathbf{z} \in \Omega_{1}
$$

and

$$
u^{2}(\mathbf{z})=-D_{k_{2}}\left[u^{2}\right](\mathbf{z})+S_{k_{2}}\left[\frac{\partial u^{2}}{\partial n}\right](\mathbf{z}), \mathbf{z} \in \Omega_{2} .
$$

Equations for the unknown boundary values of $u^{j}$ and $\frac{\partial u^{j}}{\partial n}$ on $\Gamma$ are obtained by enforcing the transmission conditions (4); once these unknown functions have been obtained the representation formulae (7) and (8) can be used to evaluate the solutions $u^{1}$ and $u^{2}$ throughout $\Omega_{1}$ and $\Omega_{2}$. In view of the use of Green's formula in conjunction with the physical quantities $u^{j}$ and $\frac{\partial u^{j}}{\partial n}$ as integral densities, the integral equations obtained in this manner are called "Direct". Other integral equations, such as the regularized integral equations introduced in this paper, are "Indirect": the corresponding unknown densities are not directly identifiable physical quantities.

In addition to Green's identities (7) and (8), exterior and interior boundary values of single and double layer potentials together with exterior and interior 
boundary values of the normal derivatives of these potentials are needed in the derivation of integral equations for transmission problems. A number of boundary integral operators thus arise for a given wavenumber $k$, including the single layer operator

$$
S_{k}[\varphi](\mathbf{x})=\int_{\Gamma} G_{k}(\mathbf{x}-\mathbf{y}) \varphi(\mathbf{y}) d s(\mathbf{y}), \mathbf{x} \text { on } \Gamma,
$$

the double layer operator $D_{k}$

$$
D_{k}[\varphi](\mathbf{x})=\int_{\Gamma} \frac{\partial G_{k}(\mathbf{x}-\mathbf{y})}{\partial n(\mathbf{y})} \varphi(\mathbf{y}) d s(\mathbf{y}), \mathbf{x} \text { on } \Gamma
$$

its adjoint $D_{k}^{*}$

$$
D_{k}^{*}[\varphi](\mathbf{x})=\int_{\Gamma} \frac{\partial G_{k}(\mathbf{x}-\mathbf{y})}{\partial n(\mathbf{x})} \varphi(\mathbf{y}) d s(\mathbf{y}), \mathbf{x} \text { on } \Gamma
$$

and the hypersingular operator

$$
\begin{aligned}
& N_{k}[\varphi](\mathbf{x})=\mathrm{FP} \int_{\Gamma} \frac{\partial^{2} G_{k}(\mathbf{x}-\mathbf{y})}{\partial n(\mathbf{x}) \partial n(\mathbf{y})} \varphi(\mathbf{y}) d s(\mathbf{y}) \\
& =k^{2} \int_{\Gamma} G_{k}(\mathbf{x}-\mathbf{y})(n(\mathbf{x}) \cdot n(\mathbf{y})) \varphi(\mathbf{y}) d s(\mathbf{y})+\operatorname{PV} \int_{\Gamma} \partial_{s} G_{k}(\mathbf{x}-\mathbf{y}) \partial_{s} \varphi(\mathbf{y}) d s(\mathbf{y}) .
\end{aligned}
$$

The operator $N_{k}$ equals the limit of the normal derivative of the double layer potential on $\Gamma$ from the outside of the domain. The expressions in (12) contain Hadamard Finite Parts integrals as well as Cauchy Principal Value integrals; the second line in this equation, further, utilizes the tangential derivative operator $\partial_{s}$ on the curve $\Gamma$.

The mapping properties of the boundary integral operators above in appropriate Sobolev spaces $H^{s}(\Gamma)$ of functions defined on the curve $\Gamma[23,38](s \in \mathbb{R})$ play central roles in determination of existence and uniqueness of solutions for the integral equations of scattering theory. The following theorem outlines relevant results in these regards. (In what follows and throughout this paper, for a complex number $z$ the symbols $\Re(z)$ and $\Im(z)$ denote the real and imaginary parts of $z$.)

Theorem 3.1 Let $k$ be a complex number satisfying $\Re k \geq 0$ and $\Im(k) \geq 0$ and assume $\Gamma$ is a smooth curve. Then for all $s \geq 0$ the boundary integral operators (9)-(12) define continuous mappings $S_{k}: H^{s-1 / 2}(\Gamma) \rightarrow H^{s+1 / 2}(\Gamma) ; N_{k}$ : $H^{s+1 / 2}(\Gamma) \rightarrow H^{s-1 / 2}(\Gamma) ; D_{k}^{*}: H^{s+1 / 2}(\Gamma) \rightarrow H^{s+7 / 2}(\Gamma) ;$ and $D_{k}: H^{s+1 / 2}(\Gamma) \rightarrow$ $H^{s+7 / 2}(\Gamma)$ for all $s \geq 0$.

Proof. The first two properties are classical [38]. The last two properties result from the increased regularity of the corresponding kernels. Indeed, assuming that the boundary curve $\Gamma$ is given by $\Gamma=\left\{x(t)=\left(x_{1}(t), x_{2}(t)\right), \quad 0 \leq\right.$ 
$t \leq 2 \pi\}$ where $x: \mathbb{R} \rightarrow \mathbb{R}^{2}$ is smooth and $2 \pi$ periodic, the operators $D_{k}^{*}$ can be expressed [23] in the parametric form

$$
\left(D_{k}^{*} \varphi\right)(x(t))=\frac{1}{\left|x^{\prime}(t)\right|} \int_{0}^{2 \pi} L(t, \tau) \varphi(x(\tau)) d \tau
$$

where, letting $J_{1}$ denote the first order Bessel function of the first kind, the kernel $L(t, \tau)$ is given by

$$
\begin{aligned}
& L(t, \tau)=L_{1}(t, \tau)+ \\
& \frac{k}{4 \pi} \frac{J_{1}(k|x(t)-x(\tau)|)}{|x(t)-x(\tau)|} \frac{(x(t)-x(\tau)) \cdot n(t)}{|x(t)-x(\tau)|^{2}}|x(t)-x(\tau)|^{2} \log \left(4 \sin ^{2} \frac{t-\tau}{2}\right)
\end{aligned}
$$

for a certain smooth function $L_{1}$ of the variables $t$ and $\tau,(t, \tau) \in[0,2 \pi] \times$ $[0,2 \pi]$. It is easy to check that for smooth curves $\Gamma$ the kernel $L$ is a $C^{1}$ function of the variables $t$ and $\tau$ for $(t, \tau) \in[0,2 \pi] \times[0,2 \pi]$ whose second derivative has a logarithmic singularity. Thus, $D_{k}^{*}$ is regularizing of three orders, that is $D_{k}^{*}: H^{s+1 / 2}(\Gamma) \rightarrow H^{s+7 / 2}(\Gamma)$. Similar considerations lead to the result about the mapping properties of the operator $D_{k}$.

In addition to the mapping properties laid down in Theorem 3.1, below in this paper we also utilize additional smoothing properties presented in Theorem 3.2 that arise as certain differences of boundary integral operators are considered.

Theorem 3.2 Let two wavenumbers $\kappa_{1}$ and $\kappa_{2}$ such that $\Re \kappa_{j} \geq 0$ and $\Im\left(\kappa_{j}\right) \geq$ 0 be given. Then, the operators $S_{\kappa_{1}}-S_{\kappa_{2}}$ and $N_{\kappa_{1}}-N_{\kappa_{2}}$ are regularizing operators by three orders and by one order, respectively. More precisely, for all $s \geq 0$ these operator differences define continuous mappings $S_{\kappa_{1}}-S_{\kappa_{2}}: H^{s-1 / 2}(\Gamma) \rightarrow$ $H^{s+5 / 2}(\Gamma)$ and $N_{\kappa_{1}}-N_{\kappa_{2}}: H^{s+1 / 2}(\Gamma) \rightarrow H^{s+3 / 2}(\Gamma)$.

Proof. Lemma 10 in [30] provides a proof for the single layer case in a slightly different setting. The corresponding result for the hypersingular operator may be established in a similar manner (the difference $N_{\kappa_{1}}-N_{\kappa_{2}}$ can be expressed as an operator with a weakly singular kernel) or, alternatively, from the result for $S_{\kappa_{1}}-S_{\kappa_{2}}$ together with the relation

$$
\left(N_{\kappa_{1}}-N_{\kappa_{2}}\right) S_{\kappa_{1}}=\left(D_{\kappa_{1}}^{*}\right)^{2}-N_{\kappa_{2}}\left(S_{\kappa_{1}}-S_{\kappa_{2}}\right)-\left(D_{\kappa_{2}}^{*}\right)^{2}
$$

which follows directly from the Calderón relation [33].

Remark 3.3 For improved readability in what follows we rely on a slight notational abuse: the boundary integral operators associated with the wavenumbers $k_{1}$ and $k_{2}$ of regions $\Omega_{1}$ and $\Omega_{2}$ will be denoted by means of a subindex 1 or 2, respectively, i.e., $S_{1}=S_{k_{1}}, S_{2}=S_{k_{2}}$ and, further, $D_{j}=D_{k_{j}}, D_{j}^{*}=D_{k_{j}}^{*}$ and $N_{j}=N_{k_{j}}$ for $j=1,2$. This notation is reserved for the wavenumbers $k_{1}$ and $k_{2}$ exclusively, however: for other wavenumbers $k=\kappa_{1}$ to be considered 
the full wavenumber dependence will be displayed as part of the notation of the boundary integral operators - e.g. the single layer operators with wavenumber $k=\kappa_{1}$ will be denoted by $S_{\kappa_{1}}$, etc; the notation $S_{1}$ is reserved for the operator $S_{k_{1}}$.

As indicated earlier in this section, utilizing the Green identities (7) and (8) to represent the fields in the regions $\Omega_{1}$ and $\Omega_{2}$, respectively, integral equations for the problem (2)-(5) are obtained as the transmission conditions (4) are enforced on the corresponding functions $u^{1}$ and $u^{2}$ (cf. [19]). Such equations can be obtained by combining the Green's identities (7) and (8) together with corresponding Green's formulae for the incident fields (which in view of the assumption (1) satisfy the Helmholtz equation throughout space), and subsequently evaluating the interior and exterior boundary values on $\Gamma$ of the fields $u^{j}, j=1,2$ and their normal derivatives. Two pairs of integral equations thus result, one pair arising as limits are taken from the interior of $\Gamma$, and the other as limits are taken from the exterior of $\Gamma$. In view of the transmission conditions (4) these equations may be expressed as integrals involving the values of the total exterior field $u=u^{1}+u^{i n c}$ and its normal derivative $\frac{\partial u}{\partial n}$ on $\Gamma$. A linear combination of the inside and outside integral equations that express the total field as an integral, together with another linear combination of the integral equations that express the normal derivative of the total field as an integral, can then be laid down, in a search for a well posed, uniquely solvable Fredholm system of two equations and two unknowns for the transmission problem at hand. Particular selections of the values of the coefficients of these linear combinations, for example, yield the following two pairs of integral equations

$$
\begin{aligned}
& u(\mathbf{x})+\left(D_{2}-D_{1}\right)[u](\mathbf{x})-\left(\nu^{-1} S_{2}-S_{1}\right)\left[\frac{\partial u}{\partial n}\right](\mathbf{x})=u^{i n c}(\mathbf{x}) \\
& \frac{\nu^{-1}+1}{2}\left[\frac{\partial u}{\partial n}\right](\mathbf{x})+\left(D_{1}^{*}-\nu^{-1} D_{2}^{*}\right)\left[\frac{\partial u}{\partial n}\right](\mathbf{x})-\left(N_{1}-N_{2}\right)[u](\mathbf{x})=\frac{\partial u^{i n c}}{\partial n}(\mathbf{x}),
\end{aligned}
$$

and

$$
\begin{aligned}
& \frac{\nu^{-1}+1}{2} u(\mathbf{x})+\left(D_{2}-\nu^{-1} D_{1}\right)[u](\mathbf{x})+\nu^{-1}\left(S_{1}-S_{2}\right)\left[\frac{\partial u}{\partial n}\right](\mathbf{x})=\nu^{-1} u^{i n c}(\mathbf{x}) \\
& \frac{\nu^{-1}+1}{2} \frac{\partial u}{\partial n}(\mathbf{x})+\left(D_{1}^{*}-\nu^{-1} D_{2}^{*}\right)\left[\frac{\partial u}{\partial n}\right](\mathbf{x})-\left(N_{1}-N_{2}\right)[u](\mathbf{x})=\frac{\partial u^{i n c}}{\partial n}(\mathbf{x}),
\end{aligned}
$$

$(\mathbf{x} \in \Gamma)$, each one of which amounts to a well posed formulation of the transmission problem (2)-(5). These systems of equations are presented in [19] and [20], respectively. Note that in both of these systems of equations the hypersingular operators $N_{j}, j=1,2$ appear in the form $N_{1}-N_{2}$-which can be expressed in terms of a weakly singular kernel. Thus, given the mapping 
properties recounted in Theorems 3.1 and 3.2, the boundary integral operators in equations (14) and (15) are either compact operators from $H^{p}(\Gamma)$ to $H^{p}(\Gamma)\left(p \geq \frac{1}{2}\right)$, or appear as compact differences of operators between these spaces - and thus equations (14) and (15) are integral equations of the second kind. The unique solvability of equations (14) and (15) is established in [20].

While the formulations (14) and (15) are identical to each other in the case $\nu=1$, their properties differ significantly for $\nu \neq 1$. For instance, we have observed (see Section 5) that for some cases including high-contrast dielectrics the use of the formulation (15) gives rise to important reductions in the numbers of GMRES iterations required to reach a given tolerance, with corresponding reductions in computational times, vis-a-vis those required by (14). We attribute this phenomenon to the fact that the spectrum of the two-by-two matrix of integral operators on the left-hand-side of (15) accumulates only at the point $\frac{\nu^{-1}+1}{2}$, while the corresponding spectrum of the matrix operator on the left-hand-side (14) accumulates at two points, namely 1 and $\frac{\nu^{-1}+1}{2}$. The spectrum of the operator in (14) is therefore likely to be more widely spread in the complex plane than that of (15), thus conceivably giving rise to the observed differences in the number of iterations required by the GMRES solver to reach a given tolerance for equations (14) and (15).

Yet another linear combination of the pairs of integral equations arising from exterior and interior limits can be used to effect the cancellation of the identity terms. This procedure results in the following first kind integral equations with a positive definite principal part

$$
\begin{aligned}
& -\left(D_{1}+D_{2}\right)[u](\mathbf{x})+\left(\nu^{-1} S_{2}+S_{1}\right)\left[\frac{\partial u}{\partial n}\right](\mathbf{x})=u^{i n c}(\mathbf{x}) \\
& -\left(N_{1}+\nu N_{2}\right)[u](\mathbf{x})+\left(D_{1}^{*}+D_{2}^{*}\right)\left[\frac{\partial u}{\partial n}\right](\mathbf{x})=\frac{\partial u^{i n c}}{\partial n}(\mathbf{x})
\end{aligned}
$$

$(\mathbf{x} \in \Gamma)$. The unique solvability of the first-kind system $(16) H^{s+1 / 2}(\Gamma) \times$ $H^{s-1 / 2}(\Gamma), s \geq 0$ was established in [17].

Indirect versions of the direct second-kind and first-kind equations discussed above in this section can be obtained by expressing the fields $u^{j}, j=1,2$ as linear combinations of single and double layer potentials and manipulating the resulting interior and exterior boundary integral equations to parallel the characteristics obtained for the direct equations discussed above. The numerical properties of each resulting indirect integral equation is essentially identical to that associated with the corresponding direct-equation counterpart.

In the following section we introduce a new methodology for derivation of well posed regularized transmission integral equations; similar ideas were used recently [9] in the treatment of the problem of scattering by perfectly conduct- 
ing scatterers. As demonstrated in Section 5, the new transmission integral equations enable solution of transmission problems at reduced numbers of GMRES iterations, and correspondingly reduced computational times, over those resulting from all of the formulations discussed above in this section.

\section{Regularized integral equations for transmission problems}

\subsection{Regularizing operators I: layer-potential regularization}

In this section we derive regularized integral equations for transmission problems. To do this we consider acoustic fields $u^{1}$ and $u^{2}$ given by

$$
u^{1}(\mathbf{z})=D_{1}\left[R_{11}[a]+R_{12}[b]\right](\mathbf{z})-S_{1}\left[R_{21}[a]+R_{22}[b]\right](\mathbf{z}), \quad \mathbf{z} \in \mathbb{R}^{2} \backslash \Gamma
$$

and

$$
\begin{aligned}
u^{2}(\mathbf{z})=-D_{2}\left[R_{11}[a]\right. & \left.+R_{12}[b]-a\right](\mathbf{z}) \\
& +\nu^{-1} S_{2}\left[R_{21}[a]+R_{22}[b]-b\right](\mathbf{z}), \quad \mathbf{z} \in \mathbb{R}^{2} \backslash \Gamma,
\end{aligned}
$$

where $R_{i j}(i=1,2, j=1,2)$ are regularizing operators (acting on spaces of functions defined on $\Gamma$ ) which are to be selected in what follows and $(a, b)$ are densities defined on $\Gamma$ ). In view of the jump conditions of the various boundary layer potentials, the fields $u^{1}$ and $u^{2}$ solve the transmission problem (2)-(5) if and only if the system of integral equations

$$
\begin{aligned}
& \left(\frac{I}{2}-D_{2}+\left(D_{1}+D_{2}\right) R_{11}-\left(S_{1}+\nu^{-1} S_{2}\right) R_{21}\right)[a] \\
& +\left(\nu^{-1} S_{2}+\left(D_{1}+D_{2}\right) R_{12}-\left(S_{1}+\nu^{-1} S_{2}\right) R_{22}\right)[b]=-\left.u^{i n c}\right|_{\Gamma} \\
& \left(-\nu N_{2}+\left(N_{1}+\nu N_{2}\right) R_{11}-\left(D_{1}^{*}+D_{2}^{*}\right) R_{21}\right)[a] \\
+ & \left(\frac{I}{2}+K_{2}^{T}+\left(N_{1}+\nu N_{2}\right) R_{12}-\left(D_{1}^{*}+D_{2}^{*}\right) R_{22}\right)[b]=-\left.\left(\partial u^{i n c} / \partial n\right)\right|_{\Gamma} .
\end{aligned}
$$

is satisfied. The system of equations (19) can be expressed in the form

$$
\mathcal{A}\left(\begin{array}{l}
a \\
b
\end{array}\right)=-\left(\begin{array}{c}
\left.u^{i n c}\right|_{\Gamma} \\
\left.\left(\partial u^{i n c} / \partial n\right)\right|_{\Gamma}
\end{array}\right)
$$

in terms of a certain $2 \times 2$ matrix $\mathcal{A}$ with operator entries.

Our goal is to produce matrix operators $\mathcal{R}=\left(R_{i j}\right)_{1 \leq i, j \leq 2}$ such that the matrix operators $\mathcal{A}$ in equation (20) is (i) A compact perturbation of the a multiple 
of the identity matrix operator, and (ii) It is invertible. In addition, we strive to construct operators $\mathcal{R}$ that lend themselves to (iii) Accurate and efficient numerical implementations, and require iv) Reduced numbers of iterations when the system of equations is solved by means of the iterative linear algebra solver GMRES.

Letting $\mathcal{A}_{i j}, 1 \leq i, j \leq 2$ denote the entries of the matrix $\mathcal{A}$, we consider at first the operator $\mathcal{A}_{21}=-\nu N_{2}+\left(N_{1}+\nu N_{2}\right) R_{11}-\left(D_{1}^{*}+D_{2}^{*}\right) R_{21}$, and we seek to obtain operators $R_{11}$ and $R_{21}$ such that $\mathcal{A}_{21}$ maps $H^{s}(\Gamma)$ into $H^{s+1}(\Gamma)$. Given the smoothing properties of the operators $D_{j}^{*}(j=1,2)$, we seek at first to obtain an operator $R_{11}$ for which $-\nu N_{2}+\left(N_{1}+\nu N_{2}\right) R_{11}$ maps $H^{s}(\Gamma)$ into $H^{s+1}(\Gamma)$. It is natural to seek $R_{11}$ of the form $R_{11}=\alpha I$, so that $-\nu N_{2}+\left(N_{1}+\nu N_{2}\right) R_{11}=\alpha N_{1}-\nu(1-\alpha) N_{2}$. The latter operator has the desired property provided that $\alpha=\nu(1-\alpha)$, that is $\alpha=\frac{\nu}{1+\nu}$. We then seek an operator $R_{21}$ for which $\mathcal{A}_{11}=\frac{I}{2}-D_{2}+\left(D_{1}+D_{2}\right) R_{11}-\left(S_{1}+\nu^{-1} S_{2}\right) R_{21}$ is a compact perturbation of the identity operator. Given the selection made above for the operator $R_{11}$ and the smoothing properties of the operators $D_{j}^{*}$, we need to obtain an operator $\mathcal{R}_{21}$ such that $\left(S_{1}+\nu^{-1} S_{2}\right) R_{21}=-\frac{I}{2}+R$ for some continuous operator $R: H^{s}(\Gamma) \rightarrow H^{s+1}(\Gamma)$. It is natural to seek for operators of the form $R_{21}=\beta N_{\kappa_{1}}, \kappa_{1} \in \mathbb{C}$. (We emphasize here that use of a wavenumber $\kappa_{1}$ which is different from that inherent in the given transmission problem and that, indeed, may be taken to be complex, plays a crucial role to ensure invertibility of the resulting systems of equations; see Section 4.2.) We have that $\beta\left(S_{1}+\nu^{-1} S_{2}\right) N_{\kappa_{1}}=\beta S_{1}\left(N_{\kappa_{1}}-N_{1}\right)+\beta \nu^{-1} S_{2}\left(N_{\kappa_{1}}-\right.$ $\left.N_{2}\right)-\frac{\beta\left(1+\nu^{-1}\right)}{4} I+\beta D_{1}^{2}+\beta \nu^{-1} D_{2}^{2}$, and thus we see that $\beta=\frac{2 \nu}{1+\nu}$ would render operators with the desired property. We require next that the operator $\mathcal{A}_{22}=$ $\frac{I}{2}+D_{2}^{*}+\left(N_{1}+\nu N_{2}\right) R_{12}-\left(D_{1}^{*}+D_{2}^{*}\right) R_{22}$ is a compact perturbation of the identity operator. Similar considerations lead to the choice $R_{12}=\gamma S_{\kappa_{1}}$ where $\gamma=-\frac{2}{1+\nu}$. Given that the choices presented above lead to $R_{21}=2 N_{\kappa_{1}} R_{11}$, it is natural to choose $R_{22}=\frac{1}{1+\nu} I$.

In conclusion, the matrix operator $\mathcal{R}$ takes on the form

$$
\begin{aligned}
& R_{11}=\frac{\nu}{1+\nu} I \\
& R_{12}=-\frac{2}{1+\nu} S_{\kappa_{1}} \\
& R_{21}=\frac{2 \nu}{1+\nu} N_{\kappa_{1}} \\
& R_{22}=\frac{1}{1+\nu} I,
\end{aligned}
$$

where $\kappa_{1}$ is a wavenumber such that $\Re\left(\kappa_{1}\right) \geq 0$ and $\Im\left(\kappa_{1}\right) \geq 0$. The following theorem establishes that the matrix operator that enters the integral equation formulation (19) with regularizing operator $\mathcal{R}$ given in equation (21) is a compact perturbation of the identity matrix. 
Theorem 4.1 Let $\mathcal{A}$ be the operator in the left-hand-side of equation (19), where $\mathcal{R}$ is given by equation (21). Then the operator $\mathcal{A}$ is a compact perturbation of the identity matrix in the space $H^{s}(\Gamma) \times H^{s}(\Gamma)$ for all $s \geq \frac{1}{2}$.

Proof. For the 1,1 component of the matrix operator $\mathcal{A}$, which is given by

$$
\begin{aligned}
\mathcal{A}_{11} & =\frac{I}{2}-\frac{2 \nu}{1+\nu}\left(S_{1}+\nu^{-1} S_{2}\right) N_{\kappa_{1}}-D_{2}+\frac{\nu}{1+\nu}\left(D_{1}+D_{2}\right) \\
& =\frac{I}{2}-2 S_{\kappa_{1}} N_{\kappa_{1}}-\frac{2 \nu}{1+\nu}\left[\left(S_{1}-S_{\kappa_{1}}\right)+\nu^{-1}\left(S_{2}-S_{\kappa_{1}}\right)\right] N_{\kappa_{1}} \\
& -D_{2}+\frac{\nu}{1+\nu}\left(D_{1}+D_{2}\right),
\end{aligned}
$$

we have $\mathcal{A}_{11}=I+\mathcal{A}_{11}^{r}$ where

$$
\begin{aligned}
\mathcal{A}_{11}^{r} & =-2\left(D_{\kappa_{1}}^{*}\right)^{2}-\frac{2 \nu}{1+\nu}\left[\left(S_{1}-S_{\kappa_{1}}\right)+\nu^{-1}\left(S_{2}-S_{\kappa_{1}}\right)\right] N_{\kappa_{1}} \\
& -D_{2}+\frac{\nu}{1+\nu}\left(D_{1}+D_{2}\right) .
\end{aligned}
$$

Given that $D_{j}: H^{s}(\Gamma) \rightarrow H^{s+3}(\Gamma), D_{\kappa_{1}}^{*}: H^{s}(\Gamma) \rightarrow H^{s+3}(\Gamma), N_{\kappa_{1}}: H^{s}(\Gamma) \rightarrow$ $H^{s-1}(\Gamma), S_{j}-S_{\kappa_{1}}: H^{s-1}(\Gamma) \rightarrow H^{s+2}(\Gamma)$ for $j=1,2$, we see that $\mathcal{A}_{11}^{r}$ : $H^{s}(\Gamma) \rightarrow H^{s+2}(\Gamma)$.

The 1,2 entry of the matrix operator $\mathcal{A}$ is given by

$$
\begin{aligned}
\mathcal{A}_{12} & =\nu^{-1} S_{2}-\frac{2}{1+\nu}\left(D_{1}+D_{2}\right) S_{\kappa_{1}}-\frac{1}{1+\nu}\left(S_{1}+\nu^{-1} S_{2}\right) \\
& =\frac{1}{1+\nu}\left(S_{2}-S_{1}\right)-\frac{2}{1+\nu}\left(D_{1}+D_{2}\right) S_{\kappa_{1}} ;
\end{aligned}
$$

which, as it is checked easily, maps $H^{s}(\Gamma)$ into $H^{s+1}(\Gamma)$.

The 2,1 entry of $\mathcal{A}$ is given by

$$
\begin{aligned}
\mathcal{A}_{21} & =-\nu N_{2}+\frac{\nu}{1+\nu}\left(N_{1}+\nu N_{2}\right)-\frac{2 \nu}{1+\nu}\left(D_{1}^{*}+D_{2}^{*}\right) N_{\kappa_{1}} \\
& =\frac{\nu}{1+\nu}\left(N_{1}-N_{2}\right)-\frac{2 \nu}{1+\nu}\left(D_{1}^{*}+D_{2}^{*}-2 D_{\kappa_{1}}^{*}\right) N_{\kappa_{1}} \\
& -\frac{4 \nu}{1+\nu} N_{\kappa_{1}} D_{\kappa_{1}} .
\end{aligned}
$$

Given that $N_{1}-N_{2}: H^{s}(\Gamma) \rightarrow H^{s+1}(\Gamma), D_{j}^{*}-D_{\kappa_{1}}^{*}: H^{s}(\Gamma) \rightarrow H^{s+3}(\Gamma)$, $N_{\kappa_{1}}: H^{s}(\Gamma) \rightarrow H^{s-1}(\Gamma), D_{\kappa_{1}}: H^{s}(\Gamma) \rightarrow H^{s+3}(\Gamma)$ for $j=1,2$, we see that $\mathcal{A}_{21}: H^{s}(\Gamma) \rightarrow H^{s+1}(\Gamma)$. 
Finally, the 2, 2 entry of $\mathcal{A}$, which is given by

$$
\begin{aligned}
\mathcal{A}_{22} & =\frac{I}{2}-\frac{2}{1+\nu}\left(N_{1}+\nu N_{2}\right) S_{\kappa_{1}}+D_{2}^{*}-\frac{1}{1+\nu}\left(D_{1}^{*}+D_{2}^{*}\right) \\
& =\frac{I}{2}-2 N_{\kappa_{1}} S_{\kappa_{1}}-\frac{2}{1+\nu}\left[\left(N_{1}-N_{\kappa_{1}}\right)+\nu\left(N_{2}-N_{\kappa_{1}}\right)\right] S_{\kappa_{1}} \\
& +D_{2}^{*}-\frac{1}{1+\nu}\left(D_{1}^{*}+D_{2}^{*}\right)
\end{aligned}
$$

can be expressed in the form $\mathcal{A}_{22}=I+\mathcal{A}_{22}^{r}$, where

$$
\begin{aligned}
\mathcal{A}_{22}^{r}=-2 D_{\kappa_{1}}^{2}-\frac{2}{1+\nu}\left[\left(N_{1}-N_{\kappa_{1}}\right)\right. & \left.+\nu\left(N_{2}-N_{\kappa_{1}}\right)\right] S_{\kappa_{1}} \\
& +D_{2}^{*}-\frac{1}{1+\nu}\left(D_{1}^{*}+D_{2}^{*}\right) .
\end{aligned}
$$

Given that $N_{j}-N_{\kappa_{1}}: H^{s}(\Gamma) \rightarrow H^{s+1}(\Gamma)$, the mapping properties of the single and double layer potentials tell us that $\mathcal{A}_{22}^{r}$ maps $H^{s}(\Gamma)$ continuously into $H^{s+2}(\Gamma)$, and the proof is complete.

4.2 Invertibility of the integral formulation (19) with $\mathcal{R}$ given by (21)

Having established that the operator $\mathcal{A}$ equals the sum of the identity operator and a compact operator we turn next to its invertibility, or what is equivalent (in view of the Fredholm theory), to its injectivity. The freedom to select complex values of the regularization wavenumber $\kappa_{1}$ plays a fundamental role in these regards.

Theorem 4.2 Let $R_{11}$ and $R_{22}$ be given as in equation (21), and let $R_{12}$ and $R_{21}$ satisfy the positivity relations

$$
\begin{aligned}
& -\Im \int_{\Gamma}\left(R_{12}[b]\right) \bar{b} d s>0 \text { for all } b \in H^{-1 / 2}(\Gamma) b \neq 0 \\
& \Im \int_{\Gamma}\left(R_{21}[a]\right) \bar{a} d s>0 \text { for all } a \in H^{1 / 2}(\Gamma) a \neq 0 .
\end{aligned}
$$

Then the operator $\mathcal{A}: H^{s}(\Gamma) \times H^{s}(\Gamma) \rightarrow H^{s}(\Gamma) \times H^{s}(\Gamma)$ is injective.

Proof. Let $(a, b)$ be a solution of equations (19) with zero right-hand side and let $u^{1}$ and $u^{2}$ be given by equations (17) and (18). In view of classical uniqueness results for transmission problems we see that $u_{1}=0$ in $\Omega_{1}$ and $u_{2}=0$ in $\Omega_{2}$. It follows that

$$
u_{+}^{1}=\partial u_{+}^{1} / \partial n=0 \quad \text { and } \quad u_{-}^{2}=\partial u_{-}^{2} / \partial n=0 \quad \text { on } \quad \Gamma
$$


where the subscript + (resp. the subscript - ) denotes boundary values that are obtained as $\Gamma$ is approached from the exterior domain $\Omega_{1}$ (resp. from the interior domain $\Omega_{2}$ ). Noting that equations (17) and (18) define $u^{1}$ and $u^{2}$ everywhere outside $\Gamma$, we may also evaluate the limits of $u^{1}$ (resp. $u^{2}$ ) as $\Gamma$ is approached from the interior domain $\Omega_{2}$ (resp. the exterior domain $\Omega_{1}$ ). Using the relations (28) together with the well known jump formulas for the various layer potentials we thus obtain

$$
\begin{aligned}
u_{-}^{1} & =-R_{11}[a]-R_{12}[b], \partial u_{-}^{1} / \partial n=-R_{21}[a]-R_{22}[b] \\
u_{+}^{2}=a-R_{11}[a]-R_{12}[b], \partial u_{+}^{2} / \partial n & =\nu^{-1}\left(b-R_{21}[a]-R_{22}[b]\right),
\end{aligned}
$$

and, therefore,

$$
\begin{aligned}
\nu \int_{\Gamma} u_{+}^{2} \overline{\partial u_{+}^{2} / \partial n} & d s=\int_{\Gamma} a \bar{b} d s-\int_{\Gamma} a \overline{R_{21}[a]} d s-\int_{\Gamma} a \overline{R_{22}[b]} d s \\
& -\int_{\Gamma}\left(R_{11}[a]\right) \bar{b} d s-\int_{\Gamma}\left(R_{12}[b]\right) \bar{b} d s+\int_{\Gamma} u_{-}^{1} \overline{\partial u_{-}^{1} / \partial n} d s .
\end{aligned}
$$

Using the definition of the operators $R_{11}$ and $R_{22}$ given in equation (21) we then obtain

$$
\begin{aligned}
\nu \int_{\Gamma} u_{+}^{2} \overline{\partial u_{+}^{2} / \partial n} d s & =-\int_{\Gamma} a \overline{R_{21}[a]} d s-\int_{\Gamma}\left(R_{12}[b]\right) \bar{b} d s \\
& +\int_{\Gamma} u_{-}^{1} \overline{\partial u_{-}^{1} / \partial n} d s
\end{aligned}
$$

which in view of the Green identity can be also expressed in the form

$$
\begin{aligned}
\nu \int_{\Gamma} u_{+}^{2} \overline{\partial u_{+}^{2} / \partial n} d s & =-\int_{\Gamma} a \overline{R_{21}[a]} d s-\int_{\Gamma}\left(R_{12}[b]\right) \bar{b} d s \\
& +\int_{\Omega_{2}}\left(-k_{1}^{2}\left|u^{1}\right|^{2}+\left|\nabla u^{1}\right|^{2}\right) d x .
\end{aligned}
$$

Taking the imaginary part of this equation we obtain

$$
\nu \Im \int_{\Gamma} u_{+}^{2} \overline{\partial u_{+}^{2} / \partial n} d s=\Im \int_{\Gamma}\left(R_{21}[a]\right) \bar{a} d s-\Im \int_{\Gamma}\left(R_{12}[b]\right) \bar{b} d s
$$

and, thus, in view of equations (26) and (27)

$$
\Im \int_{\Gamma} u_{+}^{2} \overline{\partial u_{+}^{2} / \partial n} d s \geq 0 .
$$

Since $u^{2}$ is a radiative solution of the Helmholtz equation with wavenumber $k_{2}$ in the domain $\Omega_{1}$ it follows $\left[16\right.$, p. 78] that $u^{2}=0$ in $\Omega_{1}$, and, thus, $\Im \int_{\Gamma}\left(R_{21}[a]\right) \bar{a} d s=0$ and $\Im \int_{\Gamma}\left(R_{12} b\right) \bar{b} d s=0$. Invoking once again equations (26) and (27), finally, we obtain $a=b=0$ on $\Gamma$. It follows that the operator $\mathcal{A}$ is injective, as claimed. 
Theorem 4.3 Let $\mathcal{R}$ be defined as in equations (21) and let $\kappa_{1}$ be given by

$$
\kappa_{1}=\kappa+i \varepsilon, \quad \text { with } \kappa>0 \text { and } \varepsilon>0 .
$$

Then the operator $\mathcal{A}: H^{s}(\Gamma) \times H^{s}(\Gamma) \rightarrow H^{s}(\Gamma) \times H^{s}(\Gamma)$ is invertible for all $s \geq \frac{1}{2}$.

Proof. Given the definitions (21) of the operators $R_{12}$ and $R_{21}$ and the results in Appendix A (Lemma A.1 and Lemma A.2), we see that the operators $R_{12}$ and $R_{21}$ satisfy the positivity relations (26) and (27) respectively. It therefore follows from Theorem 4.2 that the operator $\mathcal{A}$ is injective. In view of Theorem 4.1 the claimed invertibility follows from the Fredholm theory and the proof is thus complete.

\subsection{Regularizing operators II: Principal symbol regularization}

In this section we introduce an alternative choice of regularizing operators $\mathcal{R}$ that results as the operators in equation (21) are replaced by operators that, for a given parametrization of the underlying curve $\Gamma$, have the same leading order asymptotics in Fourier space. In what follows we assume, without loss of generality, that $\Gamma$ is represented by means of a smooth and $2 \pi$ periodic parametrization $x:[0,2 \pi] \rightarrow \Gamma$.

For a given $\kappa>0$ and $\varepsilon>0$ let us define the following functions

$$
p^{N}(\xi ; \kappa+i \varepsilon)=-\frac{1}{2} \sqrt{|\xi|^{2}-(\kappa+i \varepsilon)^{2}}, p^{S}(\xi ; \kappa+i \varepsilon)=\frac{1}{2 \sqrt{|\xi|^{2}-(\kappa+i \varepsilon)^{2}}}
$$

for real values of the argument $\xi$. The square root featured in equation (33) is defined so that $\Im\left(p^{N}(\xi ; \kappa+i \varepsilon)\right)>0$ and $\Im\left(p^{S}(\xi ; \kappa+i \varepsilon)\right)>0$ for all $\xi$. Using these functions together with the given parametrization $x=x(t)$ of the curve $\Gamma$ we define (cf. [38]) operators $\sigma_{\kappa+i \varepsilon}^{N, x}$ and $\sigma_{\kappa+i \varepsilon}^{S, x}$ that are Fourier multipliers with symbols $p^{N}(\xi ; \kappa+i \varepsilon)$ and $p^{S}(\xi ; \kappa+i \varepsilon)$ respectively: given elements $\phi \in H^{s+1 / 2}(\Gamma)$ and $\psi \in H^{s-1 / 2}(\Gamma)$ and letting $\hat{\phi}(n)$ and $\hat{\psi}(n)$ denote the Fourier coefficients of the functions $\phi(x(t))$ and $\psi(x(t))\left|x^{\prime}(t)\right|$, respectively, we define

$$
\sigma_{\kappa+i \varepsilon}^{N, x}[\phi](x(t))=\frac{1}{\left|x^{\prime}(t)\right|} \sum_{n \in \mathbb{Z}} p^{N}(n ; \kappa+i \varepsilon) \hat{\phi}(n) e^{i n t}
$$

and

$$
\sigma_{\kappa+i \varepsilon}^{S, x}[\psi](x(t))=\sum_{n \in \mathbb{Z}} p^{S}(n ; \kappa+i \varepsilon)(n) \hat{\psi}(n) e^{i n t} .
$$

It is easy to check that, denoting by $H^{s}[0,2 \pi]$ the order- $s$ Sobolev space of $2 \pi$-periodic functions in the real line [23], $\sigma_{\kappa+i \varepsilon}^{N, x}$ and $\sigma_{\kappa+i \varepsilon}^{S, x}$ define continuous maps $\sigma_{\kappa+i \varepsilon}^{N, x}: H^{r+1 / 2}[0,2 \pi] \rightarrow H^{r-1 / 2}[0,2 \pi]$ and $\sigma_{\kappa+i \varepsilon}^{S, x}: H^{r-1 / 2}[0,2 \pi] \rightarrow$ 
$H^{r+1 / 2}[0,2 \pi]$. Further, these operators possess the following positivity relations

$\Im \int_{0}^{2 \pi} \sigma_{\kappa+i \varepsilon}^{N, x}[\phi](x(t)) \bar{\phi}(x(t))\left|x^{\prime}(t)\right| d t>0 \quad$ for every $\quad \phi \in H^{1 / 2}[0,2 \pi], \phi \neq 0$

and

$\Im \int_{0}^{2 \pi} \sigma_{\kappa+i \varepsilon}^{S, x}[\psi](x(t)) \bar{\psi}(x(t))\left|x^{\prime}(t)\right| d t>0$ for every $\quad \psi \in H^{-1 / 2}[0,2 \pi], \quad \psi \neq 0$

which follow from the formulae

$$
\Im \int_{0}^{2 \pi} \sigma_{\kappa+i \varepsilon}^{N, x}[\phi](x(t)) \bar{\phi}(x(t))\left|x^{\prime}(t)\right| d t=\sum_{n \in \mathbb{Z}} \Im\left(p^{N}(n ; \kappa+i \varepsilon)\right)|\hat{\phi}(n)|^{2}
$$

and

$$
\Im \int_{0}^{2 \pi} \sigma_{\kappa+i \varepsilon}^{S, x}[\psi](x(t)) \bar{\psi}(x(t))\left|x^{\prime}(t)\right| d t=\sum_{n \in \mathbb{Z}} \Im\left(p^{S}(n ; \kappa+i \varepsilon)\right)|\hat{\psi}(n)|^{2}
$$

since, by definition, $\Im\left(p^{N}(n ; \kappa+i \varepsilon)\right)>0$ and $\Im\left(p^{S}(n ; \kappa+i \varepsilon)\right)>0$ for all $n \in \mathbb{Z}$.

Using the principal symbol operators $\sigma_{\kappa+i \varepsilon}^{N, x}$ and $\sigma_{\kappa+i \varepsilon}^{S, x}$ we now define a $2 \times$ 2 regularizing operator $\mathcal{R}^{x}$ that provides an alternative to the regularizing operator defined in equation (21). The entries of the new regularizing operator $\mathcal{R}^{x}$ are given by

$$
\begin{aligned}
R_{11}^{x} & =\frac{\nu}{1+\nu} I \\
R_{12}^{x} & =-\frac{2}{1+\nu} \sigma_{\kappa_{1}}^{S, x} \\
R_{21}^{x} & =\frac{2 \nu}{1+\nu} \sigma_{\kappa_{1}}^{N, x} \\
R_{22}^{x} & =\frac{1}{1+\nu} I,
\end{aligned}
$$

where $\kappa_{1}=\kappa+i \varepsilon$. As shown in what follows, use of this regularizing operator instead of $\mathcal{R}$ in equation (19) also leads to uniquely solvable integral equation formulations of the second kind.

In order to state our unique solvability result it is necessary to use parametric representations of all the integral operators in equation (19). These are given by equation (13) for the operator $D_{k}^{*}$, with a similar expression for the operator $D_{k}$, and by the expression $S_{j}^{x}[\phi](x(t))=\frac{i}{4} \int_{0}^{2 \pi} H_{0}^{(1)}\left(k_{j} \mid x(t)-\right.$ $x(\tau) \mid) \phi(x(\tau))\left|x^{\prime}(\tau)\right| d \tau$ for the single layer potential. The corresponding para- 
metric expression for the hypersingular operator $N_{j}$ is given by [22]

$$
N_{j}^{x}[\psi](x(t))=\frac{1}{4 \pi\left|x^{\prime}(t)\right|} \int_{0}^{2 \pi} \cot \frac{\tau-t}{2} \psi^{\prime}(\tau) d \tau+\frac{1}{\left|x^{\prime}(t)\right|} \int_{0}^{2 \pi} M_{j}(t, \tau) \psi(\tau) d \tau
$$

where $\psi(\tau)=\psi(x(\tau))$ and where the kernels $M_{j}(t, \tau)$ are defined as

$$
M_{j}(t, \tau)=\frac{i}{4} k_{j}^{2} H_{0}^{(1)}(k|x(t)-x(\tau)|) x^{\prime}(t) \cdot x^{\prime}(\tau)-\tilde{M}_{j}(t, \tau)
$$

with

$$
\tilde{M}_{j}(t, \tau)=\frac{\partial^{2}}{\partial t \partial \tau}\left\{\frac{i}{4} H_{0}^{(1)}\left(k_{j}|x(t)-x(\tau)|\right)+\frac{1}{4 \pi} \ln \left(4 \sin ^{2} \frac{t-\tau}{2}\right)\right\} .
$$

As indicated above and established in the following theorem, the system (19) regularized by means of the principal symbol operators (38) indeed do form a uniquely solvable system of equations.

Theorem 4.4 Let $\kappa_{1}=\kappa+i \varepsilon$ where $\kappa>0$ and $\varepsilon>0$. The integral equations

$$
\left(\begin{array}{ll}
\mathcal{B}_{11} & \mathcal{B}_{12} \\
\mathcal{B}_{21} & \mathcal{B}_{22}
\end{array}\right)\left(\begin{array}{l}
a^{1} \\
b^{1}
\end{array}\right)=-\left(\begin{array}{c}
\left.u^{i n c}\right|_{\Gamma} \\
\left.\left(\partial u^{i n c} / \partial n\right)\right|_{\Gamma}
\end{array}\right)
$$

where

$$
\begin{aligned}
& \mathcal{B}_{11}=\frac{I}{2}+\frac{\nu}{1+\nu} D_{1}^{x}-\frac{1}{1+\nu} D_{2}^{x}-\frac{2 \nu}{1+\nu}\left(S_{1}^{x}+\nu^{-1} S_{2}^{x}\right) \sigma_{\kappa_{1}}^{N, x} \\
& \mathcal{B}_{12}=\frac{1}{1+\nu}\left(S_{2}^{x}-S_{1}^{x}\right)-\frac{2}{1+\nu}\left(D_{1}^{x}+D_{2}^{x}\right) \sigma_{\kappa_{1}}^{S, x} \\
& \mathcal{B}_{21}=\frac{\nu}{1+\nu}\left(N_{1}^{x}-N_{2}^{x}\right)-\frac{2 \nu}{1+\nu}\left(D_{1}^{*, x}+D_{2}^{*, x}\right) \sigma_{\kappa_{1}}^{N, x} \\
& \mathcal{B}_{22}=\frac{I}{2}+\frac{\nu}{1+\nu} D_{2}^{*, x}-\frac{1}{1+\nu} D_{1}^{*, x}-\frac{2}{1+\nu}\left(N_{1}^{x}+\nu N_{2}^{x}\right) \sigma_{\kappa_{1}}^{S, x}
\end{aligned}
$$

are uniquely solvable in $H^{s}[0,2 \pi] \times H^{s}[0,2 \pi]$ for all $s \geq \frac{1}{2}$. Furthermore, the matrix operator

$$
\mathcal{B}=\left(\begin{array}{ll}
\mathcal{B}_{11} & \mathcal{B}_{12} \\
\mathcal{B}_{21} & \mathcal{B}_{22}
\end{array}\right): H^{s}[0,2 \pi] \times H^{s}[0,2 \pi] \rightarrow H^{s}[0,2 \pi] \times H^{s}[0,2 \pi]
$$

is a compact perturbation of the identity matrix.

Proof. The proof follows from use of two increased regularity results. The first of these, which is established in appendix A (see also [8] for a similar 
result), establishes that for all $s \geq \frac{1}{2}$

"The operator $\left(S_{\kappa_{1}}^{x}-\sigma_{\kappa_{1}}^{S, x}\right)$ maps $H^{s}[0,2 \pi]$ continuously into $H^{s+3}[0,2 \pi]$ ".

To continue with our proof, we produce a corresponding result for the hypersingular operator $N_{\kappa_{1}}^{x}$. To do this we use the Calderón identities [33] and we obtain

$$
\left(N_{\kappa_{1}}^{x}-\sigma_{\kappa_{1}}^{N, x}\right) S_{\kappa_{1}}^{x}=-\frac{I}{4}+\left(D_{\kappa_{1}}^{x}\right)^{2}-\sigma_{\kappa_{1}}^{N, x} \sigma_{\kappa_{1}}^{S, x}-\sigma_{\kappa_{1}}^{N, x}\left(S_{\kappa_{1}}^{x}-\sigma_{\kappa_{1}}^{S, x}\right) .
$$

But, it follows immediately from $(33)$ that $p^{N}\left(\xi ; \kappa_{1}\right) p^{S}\left(\xi ; \kappa_{1}\right)=-\frac{1}{4}$, and, thus, from (34) and (35) we obtain $\sigma_{\kappa_{1}}^{N, x} \sigma_{\kappa_{1}}^{S, x}=-\frac{I}{4}$. It therefore follows that

$$
\left(N_{\kappa_{1}}^{x}-\sigma_{\kappa_{1}}^{N, x}\right) S_{\kappa_{1}}^{x}=\left(D_{\kappa_{1}}^{x}\right)^{2}-\sigma_{\kappa_{1}}^{N, x}\left(S_{\kappa_{1}}^{x}-\sigma_{\kappa_{1}}^{S, x}\right)
$$

from which, given the regularizing property of the operator $S_{\kappa_{1}}^{x}-\sigma_{\kappa_{1}}^{S, x}$, it follows that for all $s \geq \frac{1}{2}$

"The operator $\left(N_{\kappa_{1}}^{x}-\sigma_{\kappa_{1}}^{N, x}\right)$ maps $H^{s}[0,2 \pi]$ continuously into $H^{s+1}[0,2 \pi]$ ".

Taking into account the regularizing properties of the operators $S_{\kappa_{1}}^{x}-\sigma_{\kappa_{1}}^{S, x}$ and $N_{\kappa_{1}}^{x}-\sigma_{\kappa_{1}}^{N, x}$ we therefore see that the following are continuous mappings:

$$
\begin{aligned}
& \mathcal{B}_{11}-\mathcal{A}_{11}=-\frac{2 \nu}{1+\nu}\left(S_{1}^{x}+\nu^{-1} S_{2}^{x}\right)\left(\sigma_{\kappa_{1}}^{N, x}-N_{\kappa_{1}}^{x}\right): H^{s}[0,2 \pi] \rightarrow H^{s+2}[0,2 \pi] \\
& \mathcal{B}_{12}-\mathcal{A}_{12}=-\frac{2}{1+\nu}\left(D_{1}^{x}+D_{2}^{x}\right)\left(\sigma_{\kappa_{1}}^{S, x}-S_{\kappa_{1}}^{x}\right): H^{s}[0,2 \pi] \rightarrow H^{s+6}[0,2 \pi] \\
& \mathcal{B}_{21}-\mathcal{A}_{21}=-\frac{2 \nu}{1+\nu}\left(D_{1}^{*, x}+D_{2}^{*, x}\right)\left(\sigma_{\kappa_{1}}^{N, x}-N_{\kappa_{1}}^{x}\right): H^{s}[0,2 \pi] \rightarrow H^{s+4}[0,2 \pi] \\
& \mathcal{B}_{22}-\mathcal{A}_{22}=-\frac{2}{1+\nu}\left(N_{1}^{x}+\nu N_{2}^{x}\right)\left(\sigma_{\kappa_{1}}^{S, x}-S_{\kappa_{1}}^{x}\right): H^{s}[0,2 \pi] \rightarrow H^{s+2}[0,2 \pi]
\end{aligned}
$$

for all $s \geq \frac{1}{2}$. Thus, in view of Theorem 4.1 we see that $\mathcal{B}$ is a compact perturbation of the identity matrix in the space $H^{s}[0,2 \pi] \times H^{s}[0,2 \pi]$. Given the positivity relations (37) and (36) for the operators $R_{12}^{x}$ and $R_{21}^{x}$, further, it follows from Theorem 4.2 that the operator $\mathcal{B}$ is injective. The claimed invertibility of $\mathcal{B}$ therefore results from the Fredholm theory, and the proof is thus complete.

\section{$5 \quad$ Numerical results: Nyström discretizations}

To demonstrate the properties of the integral equations introduced in this paper vis-a-vis those provided by classical equations, we introduced high-order 


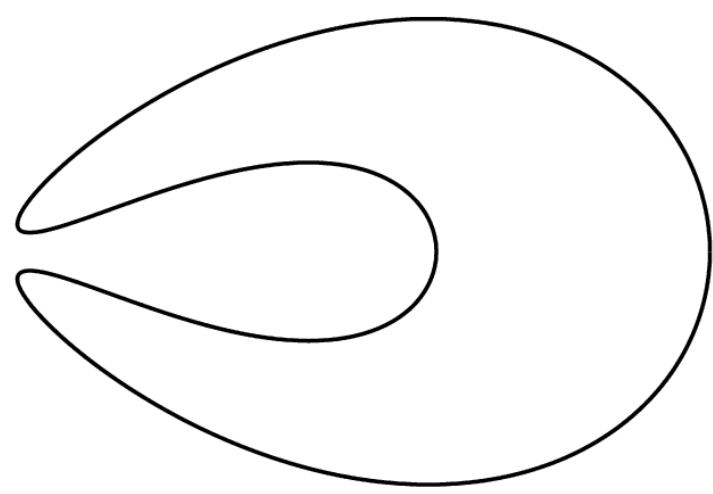

Fig. 1. Penetrable scatterer of diameter 2 for which the outer region $\Omega_{1}$ contains a cavity-like structure.

Nyström numerical implementations for all of these formulations. Our integral algorithms are based on the methodologies put forth in $[22,25,31]$ for boundary integral operators with real wavenumbers and their extensions to complex wavenumbers introduced in $[8,10]$. These methods, which are based on use of global trigonometric representation of integral densities together with explicit quadrature for products of oscillatory exponentials with weakly singular and singular kernels, will not discussed here at any length. The implementation of the principal symbol operators introduced in Section 4.3, in turn, is straightforward: it amounts to adequate uses of Fast Fourier Transforms and application of diagonal operators in Fourier space [8].

In this section we present a variety of numerical results that demonstrate the properties of the various formulations. Solutions of the linear systems arising from the Nyström discretizations were obtained in all cases by means of the fully complex un-restarted version of the iterative solver GMRES [37]. The values used in each case for the complex wavenumber $\kappa_{1}$ that enters the regularizing operators (21) and (38), which were selected by means of numerical experimentation to minimize the number of iterations required by the GMRES iterative solver, are presented in the captions of the various tables.

We present scattering experiments concerning the following three smooth scatterers: (a) a unit circle, (b) a kite-shaped scatterer given by $x(t)=(\cos t+$ $0.65 \cos 2 t-0.65,1.5 \sin t$ ) [22], and (c) a cavity whose parametrization is given by $x(t)=\left(x_{1}(t), x_{2}(t)\right), x_{1}(t)=(\cos t+2 \cos 2 t) / 2.5, x_{2}(t)=Y(t) / 2-$ $Y_{s}(t) / 48$, where $Y(t)=\sin t+\sin 2 t+1 / 2 \sin 3 t, Y_{s}(t)=-4 \sin t+7 \sin 2 t-$ $6 \sin 3 t+2 \sin 4 t$; see Figure 1. We note that each of these geometries has a diameter equal to 2. For all of our experiments we assumed plane-wave incidence $u^{\text {inc: }}$ vertical incidence in the direction of the negative $y$-axis for the circular geometry, $45^{\circ}$ incidence pointing into the fourth quadrant for the kite geometry, and horizontal incidence, pointing towards the positive $x$-axis, for the cavity structure. 
In each case our tables display maximum errors in the far-field amplitudes. The far-field amplitude $u_{\infty}^{1}(\hat{\mathbf{x}})$ for each scattering direction $\hat{\mathbf{x}}=\frac{\mathbf{x}}{|\mathbf{x}|}$ is given by

$$
u^{1}(\mathbf{x})=\frac{e^{i k_{1}|\mathbf{x}|}}{\sqrt{|\mathbf{x}|}}\left(u_{\infty}^{1}(\hat{\mathbf{x}})+\mathcal{O}\left(\frac{1}{|\mathbf{x}|}\right)\right),|\mathbf{x}| \rightarrow \infty
$$

the far-field error for each scattering direction and each numerical solution, in turn, was evaluated by means of the relation

$$
\varepsilon_{\infty}=\max \left|u_{\infty}^{1, \text { calc }}(\hat{\mathbf{x}})-u_{\infty}^{1, \text { ref }}(\hat{\mathbf{x}})\right|
$$

where $u_{\infty}^{1, \text { calc }}$ and $u_{\infty}^{1, \text { ref }}$ denote numerical and reference far fields, respectively. The reference far fields $u_{\infty}^{1, \text { ref }}$ were produced using the Mie-series in the case of circular geometries, and the classical formulation (15) with adequately refined discretizations and GMRES tolerance set to $10^{-12}$ for all other geometries. The maximum of the far field errors displayed on our tables were evaluated through maximization of the far-field error for a finite but sufficiently large number of scattering directions $\hat{\mathbf{x}}$.

Besides far field errors, the tables presented in this section display the numbers of iterations required by the GMRES solver to reach relative residuals that are specified in each case. Denoting the underlying frequency by $\omega$ (so that the wavenumber in the $j$-th dielectric medium $j=1,2$ is given by $k_{j}=\omega \sqrt{\varepsilon_{j}}$ ) we used discretizations ranging from 4 to 10 discretization points per interior wavelength $\lambda_{2}=2 \pi / k_{2}$, for frequencies $\omega$ in the medium to the high-frequency range corresponding to acoustic scattering problems of sizes ranging from $2.5 \lambda_{2}$ to $81.6 \lambda_{2}$. In all cases we assumed the exterior domain $\Omega_{1}$ is occupied by vacuum: $\varepsilon_{1}=1$. The columns "Unknowns" in our tables display the numbers of unknowns used in each case. (Since all of the systems of integral equations used involve two unknown integral densities, the number of unknowns used in each case equals two times the number of discretization points used.) The various integral formulations considered are as follows: the Second Kind formulations (14), denoted by SK (14) and (15) (SK (15)); the First Kind formulation (16) (FK (16)), and the Second Kind Regularized formulations (19)-(21) (SKR (19)-(21)) and (19)-(38) (SKR (19)-(38)).

As it can be seen in Table 1, our transmission solvers converge with high-order accuracy. Table 2, in turn, presents the computational times required by the matrix-vector products for each of the four formulations SK (15), FK (16), SKR (19)-(21), and SKR (19)-(38) respectively. The results presented here and throughout this paper were produced by a MATLAB implementation of the Nyström discretization on a single core of a MacBookPro computer with a $2.3 \mathrm{GHz}$ Quad-core Intel i7 processor with $16 \mathrm{~GB}$ of memory. (It was explicitly checked that the MATLAB code did not resort to use of multiple cores in our implementation.) indeed only one processor. We present computational times 


\begin{tabular}{|c|c|c|c|c|c|c|c|c|c|}
\hline \multirow{2}{*}{ Geometry } & \multirow{2}{*}{ Unknowns } & \multicolumn{2}{|c|}{ SK $(15)$} & \multicolumn{2}{c|}{ FK $(16)$} & \multicolumn{2}{c|}{ SKR $(19)-(21)$} & \multicolumn{2}{c|}{ SKR $(19)-(38)$} \\
\cline { 2 - 9 } & & Iter. & $\epsilon_{\infty}$ & Iter. & $\epsilon_{\infty}$ & Iter. & $\epsilon_{\infty}$ & Iter. & $\epsilon_{\infty}$ \\
\hline Circle & 64 & 23 & $2.6 \times 10^{-2}$ & 22 & $4.0 \times 10^{-2}$ & 16 & $1.6 \times 10^{-2}$ & 16 & $1.6 \times 10^{-2}$ \\
Circle & 128 & 22 & $6.2 \times 10^{-8}$ & 22 & $6.1 \times 10^{-8}$ & 16 & $6.3 \times 10^{-8}$ & 16 & $5.7 \times 10^{-9}$ \\
\hline \hline Kite & 128 & 45 & $3.0 \times 10^{-2}$ & 47 & $6.7 \times 10^{-2}$ & 31 & $2.8 \times 10^{-2}$ & 33 & $1.9 \times 10^{-2}$ \\
Kite & 192 & 45 & $1.8 \times 10^{-4}$ & 50 & $1.6 \times 10^{-4}$ & 31 & $7.0 \times 10^{-5}$ & 32 & $7.9 \times 10^{-5}$ \\
Kite & 256 & 45 & $4.5 \times 10^{-8}$ & 56 & $1.3 \times 10^{-7}$ & 31 & $1.7 \times 10^{-7}$ & 31 & $1.7 \times 10^{-7}$ \\
\hline \hline Cavity & 128 & 57 & $9.3 \times 10^{-3}$ & 59 & $6.3 \times 10^{-2}$ & 39 & $4.8 \times 10^{-2}$ & 51 & $3.8 \times 10^{-2}$ \\
Cavity & 192 & 58 & $3.5 \times 10^{-5}$ & 64 & $1.6 \times 10^{-4}$ & 39 & $1.1 \times 10^{-4}$ & 50 & $8.9 \times 10^{-5}$ \\
Cavity & 256 & 58 & $4.1 \times 10^{-8}$ & 68 & $1.8 \times 10^{-7}$ & 39 & $1.5 \times 10^{-7}$ & 50 & $1.6 \times 10^{-7}$ \\
\hline
\end{tabular}

Table 1

High-order accuracy of the solvers used demonstrated for the three geometries: circle, kite, and cavity. For these experiments we used $\nu=1, \omega=8, \epsilon_{1}=1$, and $\epsilon_{2}=2$, with GMRES residual tolerance equal to $10^{-8}$.

for the kite geometry only, as the computational times required by the other geometries considered in this text are extremely close to those required for the kite at the same levels of discretization. As it can be seen from the results in Table 2, the computational times required by a matrix-vector product for the SK (15) and FK (16) formulations are almost identical. Notably, the computational times required by a matrix-vector product of the SKR (19)-(38) formulation are on average only 1.01 times more expensive than those required by the classical SK (15) and FK (16) formulations, whereas the computational times required by a matrix-vector product required by a matrix-vector product of the SKR (19)-(21) formulation are on average at most 1.3 times more expensive than those required by the classical SK (15) and FK (16) formulations. Given that iteration numbers required by the SKR formulation can be as small as one-half or even less than the the corresponding numbers required by the SK and FK formulations (as demonstrated by Tables 3-7) the new SKR formulations can lead to savings in computing times by a factor of two or better - especially in the important high frequency regime.

We note that preconditioners of the FK (16) formulations have been proposed in the literature [3]: denoting by $\mathcal{C}$ the matrix operator on the left-hand side of the first kind equations (16), the idea is to to use the very operator $\mathcal{C}$ as a preconditioner, since, on account of Calderón's identities [33], the operator $\mathcal{C}^{2}$ is a compact perturbation of the identity matrix operator. We have pursued this avenue, and we have observed that, although the resulting $\mathcal{C}^{2}$ formulation converge in half as many iterations than the $\mathcal{C}$-based formulations, the $\mathcal{C}^{2}$ formulations are twice as expensive per iteration. Thus, that preconditioning strategy does not give rise to any actual computing-time gains. 


\begin{tabular}{|c|c|c|c|c|c|}
\hline Geometry & Unknowns & SK (15) & FK (16) & SKR (19)-(21) & SKR (19)-(38) \\
\hline Kite & 512 & $12.99 \mathrm{sec}$ & $12.78 \mathrm{sec}$ & $16.54 \mathrm{sec}$ & $13.97 \mathrm{sec}$ \\
Kite & 1024 & $51.55 \mathrm{sec}$ & $52.34 \mathrm{sec}$ & $66.39 \mathrm{sec}$ & $52.39 \mathrm{sec}$ \\
\hline
\end{tabular}

Table 2

Computational times required by a matrix-vector product for each of the four integral equation formulations of the transmission problems considered in this text.

\begin{tabular}{|c|c|c|c|c|c|c|c|c|c|c|c|}
\hline$\omega$ & \multirow{2}{*}{$\epsilon_{1}$} & $\epsilon_{2}$ & \multirow{2}{*}{ Unknowns } & \multicolumn{2}{|c|}{ SK $(15)$} & \multicolumn{2}{c|}{ FK $(16)$} & \multicolumn{2}{c|}{ SKR $(19)-(21)$} & \multicolumn{2}{c|}{ SKR $(19)-(38)$} \\
\cline { 4 - 11 } & & & & Iter. & $\epsilon_{\infty}$ & Iter. & $\epsilon_{\infty}$ & Iter. & $\epsilon_{\infty}$ & Iter. & $\epsilon_{\infty}$ \\
\hline 16 & 1 & 2 & 256 & 37 & $7.0 \times 10^{-8}$ & 37 & $1.2 \times 10^{-7}$ & 31 & $1.6 \times 10^{-7}$ & 31 & $1.6 \times 10^{-7}$ \\
32 & 1 & 2 & 512 & 58 & $6.2 \times 10^{-8}$ & 59 & $3.7 \times 10^{-7}$ & 40 & $2.2 \times 10^{-7}$ & 40 & $3.9 \times 10^{-7}$ \\
64 & 1 & 2 & 1024 & 99 & $1.8 \times 10^{-7}$ & 99 & $4.2 \times 10^{-7}$ & 61 & $5.0 \times 10^{-7}$ & 61 & $4.3 \times 10^{-7}$ \\
\hline
\end{tabular}

Table 3

Scattering experiment for the circular geometry with $\nu=1$ and, for the SKR (19)(21) and SKR (19)-(38) formulations, $\kappa_{1}=\left(k_{1}+k_{2}\right) / 2+4 i$. GMRES residual was set to equal $10^{-8}$.

\begin{tabular}{|c|c|c|c|c|c|c|c|c|c|c|c|}
\hline$\omega$ & \multirow{2}{*}{$\epsilon_{1}$} & $\epsilon_{2}$ & \multirow{2}{*}{ Unknowns } & \multicolumn{2}{|c|}{ SK $(15)$} & \multicolumn{2}{c|}{ FK $(16)$} & \multicolumn{2}{c|}{ SKR $(19)-(21)$} & \multicolumn{2}{c|}{ SKR $(19)-(38)$} \\
\cline { 5 - 11 } & & & & Iter. & $\epsilon_{\infty}$ & Iter. & $\epsilon_{\infty}$ & Iter. & $\epsilon_{\infty}$ & Iter. & $\epsilon_{\infty}$ \\
\hline 16 & 1 & 4 & 512 & 65 & $5.0 \times 10^{-4}$ & 71 & $1.5 \times 10^{-3}$ & 42 & $1.7 \times 10^{-3}$ & 46 & $1.6 \times 10^{-3}$ \\
32 & 1 & 4 & 1024 & 93 & $3.1 \times 10^{-3}$ & 104 & $2.0 \times 10^{-3}$ & 52 & $2.6 \times 10^{-3}$ & 62 & $2.6 \times 10^{-3}$ \\
64 & 1 & 4 & 2048 & 128 & $1.1 \times 10^{-3}$ & 138 & $2.3 \times 10^{-3}$ & 64 & $1.7 \times 10^{-3}$ & 74 & $1.6 \times 10^{-3}$ \\
128 & 1 & 4 & 4096 & 167 & $1.2 \times 10^{-3}$ & 182 & $2.3 \times 10^{-3}$ & 78 & $1.6 \times 10^{-3}$ & 83 & $1.9 \times 10^{-3}$ \\
\hline
\end{tabular}

Table 4

Scattering experiments for the kite geometry with $\nu=1$, and for the SKR (19)-(21) and SKR (19)-(38) formulations, $\kappa_{1}=\left(k_{1}+k_{2}\right) / 2+i \omega / 4$. GMRES residual was set to equal $10^{-4}$.

\begin{tabular}{|c|c|c|c|c|c|c|c|c|c|c|c|}
\hline$\omega$ & \multirow{2}{*}{$\epsilon_{1}$} & $\epsilon_{2}$ & \multirow{2}{*}{ Unknowns } & \multicolumn{2}{|c|}{ SK $(15)$} & \multicolumn{2}{|c|}{ FK $(16)$} & \multicolumn{2}{c|}{ SKR $(19)-(21)$} & \multicolumn{2}{c|}{ SKR $(19)-(38)$} \\
\cline { 4 - 11 } & & & & Iter. & $\epsilon_{\infty}$ & Iter. & $\epsilon_{\infty}$ & Iter. & $\epsilon_{\infty}$ & Iter. & $\epsilon_{\infty}$ \\
\hline 16 & 1 & 4 & 512 & 111 & $8.2 \times 10^{-4}$ & 114 & $2.9 \times 10^{-3}$ & 64 & $4.9 \times 10^{-3}$ & 70 & $4.9 \times 10^{-3}$ \\
32 & 1 & 4 & 1024 & 168 & $1.2 \times 10^{-3}$ & 179 & $6.6 \times 10^{-3}$ & 91 & $4.9 \times 10^{-3}$ & 104 & $5.0 \times 10^{-3}$ \\
64 & 1 & 4 & 2048 & 266 & $1.3 \times 10^{-3}$ & 289 & $2.9 \times 10^{-3}$ & 120 & $3.8 \times 10^{-3}$ & 145 & $3.7 \times 10^{-3}$ \\
128 & 1 & 4 & 4096 & 396 & $1.7 \times 10^{-3}$ & 433 & $3.2 \times 10^{-3}$ & 157 & $3.2 \times 10^{-3}$ & 205 & $3.3 \times 10^{-3}$ \\
\hline
\end{tabular}

Table 5

Scattering experiments for the cavity geometry, $\nu=1$, and for the SKR (19)-(21) and SKR (19)-(38) formulations, $\kappa_{1}=\left(k_{1}+k_{2}\right) / 2+i \omega / 4$. GMRES residual was set to equal $10^{-4}$.

\section{Acknowledgments}

Oscar Bruno thanks NSF and AFOSR for their support during the preparation of this work. Yassine Boubendir gratefully acknowledges support from NSF 


\begin{tabular}{|c|c|c|c|c|c|c|c|c|c|c|c|}
\hline$\omega$ & $\epsilon_{1}$ & $\epsilon_{2}$ & \multirow{2}{*}{ Unknowns } & \multicolumn{2}{|c|}{ SK $(15)$} & \multicolumn{2}{|c|}{ FK $(16)$} & \multicolumn{2}{c|}{ SKR $(19)-(21)$} & \multicolumn{2}{c|}{ SKR $(19)-(38)$} \\
\cline { 5 - 11 } & & & & Iter. & $\epsilon_{\infty}$ & Iter. & $\epsilon_{\infty}$ & Iter. & $\epsilon_{\infty}$ & Iter. & $\epsilon_{\infty}$ \\
\hline 8 & 1 & 16 & 512 & 79 & $1.4 \times 10^{-3}$ & 210 & $2.4 \times 10^{-3}$ & 65 & $1.8 \times 10^{-3}$ & 66 & $2.0 \times 10^{-3}$ \\
16 & 1 & 16 & 1024 & 122 & $5.0 \times 10^{-3}$ & 283 & $6.8 \times 10^{-3}$ & 97 & $5.6 \times 10^{-3}$ & 91 & $5.5 \times 10^{-3}$ \\
32 & 1 & 16 & 2048 & 176 & $7.8 \times 10^{-3}$ & 373 & $3.0 \times 10^{-3}$ & 112 & $2.2 \times 10^{-3}$ & 109 & $1.9 \times 10^{-3}$ \\
64 & 1 & 16 & 4096 & 263 & $9.1 \times 10^{-4}$ & 497 & $3.2 \times 10^{-3}$ & 147 & $1.9 \times 10^{-3}$ & 147 & $2.6 \times 10^{-3}$ \\
128 & 1 & 16 & 8192 & 338 & $7.7 \times 10^{-4}$ & 649 & $3.0 \times 10^{-3}$ & 187 & $2.1 \times 10^{-3}$ & 187 & $2.2 \times 10^{-3}$ \\
\hline
\end{tabular}

Table 6

Scattering experiments for the kite geometry with $\nu=\epsilon_{1} / \epsilon_{2}$, and for the SKR (19)(21) and SKR (19) $-(38)$ formulations, $\kappa_{1}=\left(k_{1}+k_{2}\right) / 2+i \omega$. GMRES residual was set to equal $10^{-4}$. Through additional experiments we have determined that the formulations SK (14) require iterations numbers equal to 129, 207, 288, 318, and 393 for the cases when $\omega=8,16,32,64$ and 128, respectively.

\begin{tabular}{|c|c|c|c|c|c|c|c|c|c|c|c|}
\hline$\omega$ & $\epsilon_{1}$ & $\epsilon_{2}$ & \multirow{2}{*}{ Unknowns } & \multicolumn{2}{|c|}{ SK $(15)$} & \multicolumn{2}{c|}{ FK $(16)$} & \multicolumn{2}{c|}{ SKR $(19)-(21)$} & \multicolumn{2}{c|}{ SKR $(19)-(38)$} \\
\cline { 5 - 11 } & & & & Iter. & $\epsilon_{\infty}$ & Iter. & $\epsilon_{\infty}$ & Iter. & $\epsilon_{\infty}$ & Iter. & $\epsilon_{\infty}$ \\
\hline 8 & 1 & 16 & 512 & 114 & $1.3 \times 10^{-3}$ & 246 & $8.7 \times 10^{-3}$ & 85 & $9.0 \times 10^{-3}$ & 85 & $8.8 \times 10^{-3}$ \\
16 & 1 & 16 & 1024 & 182 & $2.7 \times 10^{-3}$ & 429 & $1.6 \times 10^{-2}$ & 148 & $1.6 \times 10^{-2}$ & 148 & $1.5 \times 10^{-3}$ \\
32 & 1 & 16 & 2048 & 341 & $3.6 \times 10^{-3}$ & 661 & $2.1 \times 10^{-2}$ & 200 & $2.1 \times 10^{-2}$ & 202 & $2.0 \times 10^{-2}$ \\
64 & 1 & 16 & 4096 & 489 & $3.1 \times 10^{-3}$ & 1094 & $2.9 \times 10^{-3}$ & 278 & $3.4 \times 10^{-3}$ & 297 & $2.1 \times 10^{-3}$ \\
128 & 1 & 16 & 8192 & 877 & $7.0 \times 10^{-4}$ & 1560 & $2.3 \times 10^{-3}$ & 397 & $1.9 \times 10^{-3}$ & 406 & $1.6 \times 10^{-3}$ \\
\hline
\end{tabular}

Table 7

Scattering experiments for the cavity geometry, $\nu=\epsilon_{1} / \epsilon_{2}$, for the SKR (19)-(21) and SKR (19)-(38) formulations, $\kappa_{1}=\left(k_{1}+k_{2}\right) / 2+i \omega$. GMRES residual was set to equal $10^{-4}$. Through additional experiments we have determined that the formulations SK (14) require iterations numbers equal to $167,290,481,663$, and 1232 respectively for the cases when $\omega=8,16,32,64$ and 128 .

through contract DMS-1016405. Catalin Turc gratefully acknowledge support from NSF through contract DMS-1008076.

\section{A Positivity of complex wavenumber operators}

In this section we establish certain positivity properties for the operators $S_{\kappa+i \varepsilon}$ and $N_{\kappa+i \epsilon}$ (cf. [33]).

Lemma A.1 Let $\kappa>0$ and $\varepsilon>0$. Then

$$
\Im \int_{\Gamma} S_{\kappa+i \varepsilon}[\phi] \bar{\phi} d s>0, \quad \text { for all } \phi \in H^{-1 / 2}(\Gamma), \phi \neq 0 .
$$

Proof. Let

$$
U(z)=S_{\kappa+i \varepsilon}[\phi](z)=\int_{\Gamma} G_{\kappa+i \varepsilon}(z-y) \phi(y) d s(y), z \in \mathbb{R}^{2} \backslash \Gamma
$$


and call $U^{+}$and $U^{-}$the restrictions of $U$ to $\Omega_{1}$ and $\Omega_{2}$, respectively. Given that $U^{-}$is a solution of the Helmholtz equation with wavenumber $\kappa+i \epsilon$ in the domain $\Omega_{2}$ we obtain

$$
\int_{\Gamma} U^{-} \overline{\partial_{n} U^{-}} d s=\int_{\Omega_{2}}\left(\left|\nabla U^{-}\right|^{2}-(\kappa-i \varepsilon)^{2}\left|U^{-}\right|^{2}\right) d x
$$

from which it follows that

$$
\Im \int_{\Gamma} U^{-} \overline{\partial_{n} U^{-}} d s=2 \kappa \varepsilon \int_{\Omega_{2}}\left|U^{-}\right|^{2} d x .
$$

Let $R>0$ be such that $\Omega_{2}$ is contained in $B_{R}=\left\{x:\|x\|_{2} \leq R\right\}$. An application of the Green's formula in the domain $\Omega_{1} \cap B_{R}$ gives

$$
\int_{\partial B_{R}} U^{+} \overline{\partial_{r} U^{+}} d s-\int_{\Gamma} U^{+} \overline{\partial_{n} U^{+}} d s=\int_{\Omega_{1} \bigcap B_{R}}\left(\left|\nabla U^{+}\right|^{2}-(\kappa-i \varepsilon)^{2}\left|U^{+}\right|^{2}\right) d x
$$

from which we obtain

$$
\Im \int_{\partial B_{R}} U^{+} \overline{\partial_{r} U^{+}} d s-\Im \int_{\Gamma} U^{+} \overline{\partial_{n} U^{+}} d s=2 \kappa \varepsilon \int_{\Omega_{1} \bigcap B_{R}}\left|U^{+}\right|^{2} d x .
$$

Given that $\varepsilon>0$ and the resulting exponential decay of $U^{+}(x)$ as $|x| \rightarrow \infty$ it follows that $\lim _{R \rightarrow \infty} \int_{\partial B_{R}} U^{+} \overline{\partial_{r} U^{+}} d s=0$, and, thus

$$
\Im \int_{\Gamma} U^{-} \overline{\partial_{n} U^{-}} d s-\Im \int_{\Gamma} U^{+} \overline{\partial_{n} U^{+}} d s=2 \kappa \varepsilon\left(\int_{\Omega_{2}}|U|^{2} d x+\int_{\Omega_{1}}|U|^{2} d x\right) .
$$

Since $U^{+}=U^{-}=S_{\kappa+i \varepsilon}[\phi]$ and $\partial_{n} U^{-}-\partial_{n} U^{+}=\phi$ on $\Gamma$ we see that

$$
\Im \int_{\Gamma} S_{\kappa+i \varepsilon}[\phi] \bar{\phi} d s=2 \kappa \varepsilon \int_{\mathbb{R}^{2}}|U|^{2} d x
$$

and the Lemma follows.

Lemma A.2 Let $\kappa>0$ and $\varepsilon>0$. Then

$\Im \int_{\Gamma} N_{\kappa+i \varepsilon}[\psi] \bar{\psi} d s>0, \quad$ for all $\psi \in H^{1 / 2}(\Gamma), \psi \neq 0$.

Proof. Let

$$
V(z)=D_{\kappa+i \varepsilon}[\psi](z)=\int_{\Gamma} \frac{\partial G_{\kappa+i \varepsilon}(z-y)}{\partial n(y)} \psi(y) d s(y), z \in \mathbb{R}^{2} \backslash \Gamma .
$$

Given that $V$ is a solution of the Helmholtz equation with wavenumber $\kappa+i \epsilon$ in the domain $\Omega_{2}$ and a radiative solution of the Helmholtz equation with wavenumber $\kappa+i \epsilon$ in the domain $\Omega_{1}$, as in the derivation of equation (A.1) we obtain

$$
\Im \int_{\Gamma} \partial_{n} V^{+} \overline{V^{+}} d s-\Im \int_{\Gamma} \partial_{n} V^{-} \overline{V^{-}} d s=2 \kappa \varepsilon\left(\int_{\Omega_{2}}|V|^{2} d x+\int_{\Omega_{1} \bigcap_{B}}|V|^{2} d x\right) .
$$


Since $\left.V^{+}\right|_{\Gamma}-\left.V^{-}\right|_{\Gamma}=\psi$ and $\partial_{n} V^{+}=\partial_{n} V^{-}=N_{\kappa+i \varepsilon}[\psi]$ on $\Gamma$ we obtain

$$
\Im \int_{\Gamma} N_{\kappa+i \varepsilon}[\psi] \bar{\psi} d s=2 \kappa \varepsilon \int_{\mathbb{R}^{2}}|V|^{2} d x
$$

from which the result of the Lemma follows.

\section{B Increased regularity of the operator $S_{\kappa+i \varepsilon}^{x}-\sigma_{\kappa+i \varepsilon}^{S, x}$}

This appendix establishes that, given a smooth $2 \pi$ periodic parametrization $x:[0,2 \pi] \rightarrow \Gamma$ of the curve $\Gamma$, the difference between the parametric form

$S_{\kappa+i \varepsilon}^{x}[\psi](x(t))=\frac{i}{4} \int_{0}^{2 \pi} H_{0}^{(1)}((\kappa+i \varepsilon)|x(t)-x(\tau)|) \psi(x(\tau))\left|x^{\prime}(\tau)\right| d \tau$ of the single layer operator and with wavenumber $\kappa_{1}=\kappa+i \varepsilon$ and the Fourier operator $\sigma_{\kappa+i \varepsilon}^{S, x}$ defined in equation (35) is a three-order smoothing operator.

Lemma B.1 For all $s \geq \frac{1}{2}$ the operator $S_{\kappa+i \varepsilon}^{x}-\sigma_{\kappa+i \varepsilon}^{S, x}$ maps $H^{s}[0,2 \pi]$ continuously into $H^{s+3}[0,2 \pi]$.

Proof. Using the small $z$ expression [1]

$$
\frac{i}{4} H_{0}^{(1)}(k|z|)=-\frac{1}{2 \pi} \log |z|+\frac{i}{4}-\frac{1}{2 \pi}\left(\log \frac{k}{2}+C\right)+\mathcal{O}\left(|z|^{2} \log |z|\right), \quad z \rightarrow 0
$$

where $C$ is Euler's constant, we see that the kernel of the operator $S_{\kappa+i \varepsilon}^{x}$ can be expressed in the form

$$
\begin{aligned}
\frac{i}{4} H_{0}^{(1)}((\kappa & +i \varepsilon)|x(t)-x(\tau)|)\left|x^{\prime}(\tau)\right| \\
& =\frac{i}{4} H_{0}^{(1)}\left((\kappa+i \varepsilon)\left|e^{i t}-e^{i \tau}\right|\right)\left|x^{\prime}(\tau)\right|+\Phi_{1}(t, \tau)+\Phi_{2}(t, \tau)
\end{aligned}
$$

where $\Phi_{1}(t, \tau)$ is a smooth function of $(t, \tau)$ and where, letting $f(t, \tau)=t-$ $\tau \bmod 2 \pi, \Phi_{2}(t, \tau)=\mathcal{O}\left(|f(t, \tau)|^{2} \log |f(t, \tau)|\right)$ as $|f(t, \tau)| \rightarrow 0$. It follows that for given $\psi \in H^{s}[0,2 \pi]$ we may write

$$
S_{\kappa+i \varepsilon}^{x}[\psi](x(t))=S_{\kappa+i \varepsilon}^{\mathbb{S}^{1}, x}[\psi](t)+S_{\Phi_{1}}^{x}[\psi](x(t))+S_{\Phi_{2}}^{x}[\psi](x(t))
$$

where

$$
S_{\kappa+i \varepsilon}^{\mathbb{S}^{1}, x}[\psi](t)=\frac{i}{4} \int_{0}^{2 \pi} H_{0}^{(1)}\left((\kappa+i \varepsilon)\left|e^{i t}-e^{i \tau}\right|\right) \psi(x(\tau))\left|x^{\prime}(\tau)\right| d \tau
$$

and

$$
S_{\Phi_{j}}^{x}[\psi](x(t))=\int_{0}^{2 \pi} \Phi_{j}(t, \tau) \psi(x(\tau)) d \tau, j=1,2
$$


Given that the kernel $\Phi_{1}(t, \tau)$ is a smooth function of $(t, \tau)$ and taking into account the behavior of the kernel $\Phi_{2}(t, \tau)$ as $|f(t, \tau)| \rightarrow 0$, we see that $S_{\Phi_{j}}^{x}: H^{s}[0,2 \pi] \rightarrow H^{s+3}[0,2 \pi], j=1,2$. It thus suffices to show that the difference $S_{\kappa+i \varepsilon}^{\mathbb{S}^{1}, x}[\psi](x(t))-S_{\kappa+i \varepsilon}^{x}[\psi](x(t))$ defines an operator that maps $H^{s}[0,2 \pi]$ continuously into $H^{s+3}[0,2 \pi]$.

To do this we use the Fourier expansion $\psi(x(\tau))\left|x^{\prime}(\tau)\right|=\sum_{n \in \mathbb{Z}} \hat{\psi}(n) e^{i n \tau}$ and we obtain

$$
\begin{aligned}
\frac{i}{4} \int_{0}^{2 \pi} H_{0}^{(1)}\left((\kappa+i \varepsilon)\left|e^{i t}-e^{i \tau}\right|\right) & \psi(x(\tau))\left|x^{\prime}(\tau)\right| d \tau= \\
& \sum_{n=-\infty}^{\infty} \hat{\psi}(n)\left(\frac{i}{4} \int_{0}^{2 \pi} H_{0}^{(1)}\left((\kappa+i \varepsilon)\left|e^{i t}-e^{i \tau}\right|\right) e^{i n \tau} d \tau\right),
\end{aligned}
$$

so that, in view of well known relations for circular scatterers [21] there follows

$$
\frac{i}{4} \int_{0}^{2 \pi} H_{0}^{(1)}\left((\kappa+i \varepsilon)\left|e^{i t}-e^{i \tau}\right|\right) e^{i n \tau} d \tau=\frac{i \pi}{2} J_{|n|}(\kappa+i \varepsilon) H_{|n|}^{(1)}(\kappa+i \varepsilon) e^{i n t} .
$$

Now, let $=s_{|n|}(\kappa+i \varepsilon)=\frac{i \pi}{2} J_{|n|}(\kappa+i \varepsilon) H_{|n|}^{(1)}(\kappa+i \varepsilon)$. Invoking the representation of the functions $J_{|n|}(\kappa+i \varepsilon)$ and $H_{|n|}^{(1)}(\kappa+i \varepsilon)$ in terms of the Bessel functions of the third kind, so that $\frac{i \pi}{2} J_{|n|}(\kappa+i \varepsilon) H_{|n|}^{(1)}(\kappa+i \varepsilon)=I_{|n|}(\varepsilon-i \kappa) K_{|n|}(\varepsilon-i \kappa)$, together with the uniform asymptotic expansions as $\nu \rightarrow \infty$ and $|\arg z| \leq$ $\frac{\pi}{2}-\delta$,

$$
\begin{aligned}
& I_{\nu}(\nu z) \sim \frac{1}{\sqrt{2 \pi \nu}} \frac{e^{\nu \mu}}{\left(1+z^{2}\right)^{\frac{1}{4}}}\left(1+u_{1}(t) \nu^{-1}+\mathcal{O}\left(\nu^{-2}\right)\right) \\
& K_{\nu}(\nu z) \sim \frac{\sqrt{\pi}}{\sqrt{2 \nu}} \frac{e^{-\nu \mu}}{\left(1+z^{2}\right)^{\frac{1}{4}}}\left(1-u_{1}(t) \nu^{-1}+\mathcal{O}\left(\nu^{-2}\right)\right)
\end{aligned}
$$

where $\mu=\sqrt{1+z^{2}}+\ln \frac{z}{1+\sqrt{1+z^{2}}}, t=\frac{1}{\sqrt{1+z^{2}}}$, and $u_{1}(t)=\frac{3 t-5 t^{3}}{24}$ (Formulae 9.7.7 and 9.7.8 in [1]) we obtain the $|n| \rightarrow \infty$ asymptotics

$$
s_{|n|}(\kappa+i \varepsilon)=\frac{1}{2 \sqrt{n^{2}+(\varepsilon-i \kappa)^{2}}}\left(1+\mathcal{O}\left(|n|^{-2}\right)\right)=p^{S}(n ; \kappa+i \varepsilon)+\mathcal{O}\left(|n|^{-3}\right) .
$$

The last identity implies that $S_{\kappa+i \varepsilon}^{\mathbb{S}^{1}, x}-\sigma_{\kappa+i \varepsilon}^{S, x}$ maps $H^{s}[0,2 \pi]$ continuously into $H^{s+3}[0,2 \pi]$, and the proof of the lemma is thus complete.

\section{References}

[1] M. Abramowitz, I. A. Stegun, Handbook of mathematical functions with formulas, graphs, and mathematical tables, vol. 55 of National Bureau of 
Standards Applied Mathematics Series, For sale by the Superintendent of Documents, U.S. Government Printing Office, Washington, D.C., 1964.

[2] F. Alouges, S. Borel, D. Levadoux, A stable well-conditioned integral equation for electromagnetism scattering, Journal of computational and applied mathematics 204 (2) (2007) 440-451.

[3] X. Antoine, Y. Boubendir, An integral preconditioner for solving the two-dimensional scattering transmission problem using integral equations, International Journal of Computer Mathematics 85 (10) (2008) 1473-1490.

[4] X. Antoine, M. Darbas, Alternative integral equations for the iterative solution of acoustic scattering problems, The Quarterly Journal of Mechanics and Applied Mathematics 58 (1) (2005) 107-128.

[5] X. Antoine, M. Darbas, Generalized combined field integral equations for the iterative solution of the three-dimensional helmholtz equation, ESAIM: Mathematical Modelling and Numerical Analysis 41 (01) (2007) 147-167.

[6] E. Bleszynski, M. Bleszynski, T. Jaroszewicz, AIM: Adaptive integral method for solving large-scale electromagnetic scattering and radiation problems, Radio Sci. 31 (5) (1996) 1225-1251.

[7] S. Borel, D. P. Levadoux, F. Alouges, A new well-conditioned integral formulation for maxwell equations in three dimensions, IEEE Transactions on Antennas and Propagation 53 (9) (2005) 2995-3004.

[8] Y. Boubendir, C. Turc, Wave-number estimates for regularized combined field boundary integral operators in acoustic scattering problems with neumann boundary conditions, IMA Journal of Numerical Analysis.

[9] O. Bruno, T. Elling, R. Paffenroth, C. Turc, Electromagnetic integral equations requiring small numbers of krylov-subspace iterations, Journal of Computational Physics 228 (17) (2009) 6169-6183.

[10] O. Bruno, T. Elling, C. Turc, Regularized integral equations and fast high-order solvers for sound-hard acoustic scattering problems, International Journal for Numerical Methods in Engineering 91 (10) (2012) 1045-1072.

[11] O. Bruno, L. Kunyansky, A fast, high-order algorithm for the solution of surface scattering problems: basic implementation, tests, and applications, J. Comput. Phys. 169 (1) (2001) 80-110.

[12] O. P. Bruno, S. K. Lintner, Second-kind integral solvers for TE and TM problems of diffraction by open arcs, Radio Science 47 (6).

[13] O. P. Bruno, S. K. Lintner, A high-order integral solver for scalar problems of diffraction by screens and apertures in three dimensional space, J. Comput. Phys. 252 (2013) 250-274.

[14] X. Claeys, R. Hiptmair, Multi-trace boundary integral formulation for acoustic scattering by composite structures, Pure Applied Math. 66 (8) (2013) 11631201. 
[15] X. Claeys, R. Hiptmair, C. Jerez-Hanckes, Multi-trace boundary integral equations, in: Direct and Inverse Problems in Wave Propagation and Applications, I. Graham, U. Langer, M. Sini, M. Melenk, 2012.

[16] D. Colton, R. Kress, Integral Equation Methods in Scattering Theory, John Wiley \& Sons, 1983.

[17] M. Costabel, E. Stephan, A direct boundary integral equation method for transmission problems, Journal of mathematical analysis and applications 106 (2) (1985) 367-413.

[18] R. Hiptmair, C. Jerez-Hanckes, Multiple traces boundary integral formulation for helmholtz transmission problems, Adv. Appl. Math. 37 (1) (2012) 39-91.

[19] R. Kittappa, R. Kleinman, Acoustic scattering by penetrable homogeneous objects, Journal of Mathematical Physics 16 (1975) 421-432.

[20] R. Kleinman, P. Martin, On single integral equations for the transmission problem of acoustics, SIAM Journal on Applied Mathematics 48 (2) (1988) $307-325$.

[21] R. Kress, Minimizing the condition number of boundary integral operators in acoustic and electromagnetic scattering, The Quarterly Journal of Mechanics and Applied Mathematics 38 (2) (1985) 323-341.

[22] R. Kress, On the numerical solution of a hypersingular integral equation in scattering theory, Journal of Computational and Applied Mathematics 61 (3) (1995) 345-360.

[23] R. Kress, Linear integral equations, vol. 82 of Applied Mathematical Sciences, 2nd ed., Springer-Verlag, New York, 1999.

[24] R. Kress, G. Roach, Transmission problems for the helmholtz equation, Journal of Mathematical Physics 19 (1978) 1433-1437.

[25] R. Kussmaul, Ein numerisches Verfahren zur Lösung des Neumannschen Aussenraumproblems für die Helmholtzsche Schwingungsgleichung, Computing 4 (3) (1969) 246-273.

[26] A. R. Laliena, M.-L. Rapún, F.-J. Sayas, Symmetric boundary integral formulations for Helmholtz transmission problems, Appl. Numer. Math. 59 (11) (2009) 2814-2823.

URL http://dx.doi.org/10.1016/j.apnum.2008.12.030

[27] D. Levadoux, Etude d'une équation intégrale adaptaeé à la resolution hautefréquance de l'equation d'Helmholtz, Thèse de doctorat de l'Université de Paris VI FranceHabilitationsscrhift.

[28] D. Levadoux, A new integral formalism for transmission problems of electromagnetism, in: 8-th International Conference on Mathematical and Numerical Aspects of Waves, Reading, UK, July 23-27, 2007, pp. 90-92. 
[29] D. P. Levadoux, F. Millot, S. Pernet, New trends in the preconditioning of integral equations of electromagnetism, in: Scientific Computing in Electrical Engineering SCEE 2008, Springer, 2010, pp. 383-394.

[30] S. K. Lintner, O. P. Bruno, A generalized Calderón formula for open-arc diffraction problems: theoretical considerations, arXiv preprint arXiv:1204.3699.

[31] E. Martensen, Über eine Methode zum räumlichen Neumannschen Problem mit einer Anwendung für torusartige Berandungen, Acta mathematica 109 (1) (1963) $75-135$.

[32] C. Müller, Foundations of the mathematical theory of electromagnetic waves, Springer Berlin, 1969.

[33] J.-C. Nédélec, Acoustic and electromagnetic equations: Integral representations for harmonic problems, vol. 144, Springer, 2001.

[34] K.-H. L. Peng, Zhen, J.-F. Lee, Computations of electromagnetic wave scattering from penetrable composite targets using a surface integral equation method with multiple traces, IEEE Transactions on Antennas and Propagation 61 (1) (2013) 256-269.

[35] V. Rokhlin, Solution of acoustic scattering problems by means of second kind integral equations, Wave Motion 5 (3) (1983) 257-272.

[36] V. Rokhlin, Diagonal forms of translation operators for the helmholtz equation in three dimensions, Applied and Computational Harmonic Analysis 1 (1) (1993) 82-93.

[37] Y. Saad, M. H. Schultz, Gmres: A generalized minimal residual algorithm for solving nonsymmetric linear systems, SIAM Journal on scientific and statistical computing 7 (3) (1986) 856-869.

[38] J. Saranen, G. Vainikko, Periodic integral and pseudodifferential equations with numerical approximation, Springer, 2002. 